\title{
SURFACES GENERATING THE EVEN PRIMAL COHOMOLOGY OF AN ABELIAN FIVEFOLD
}

\author{
JONATHAN CONDER, EDWARD DEWEY, AND ELHAM IZADI
}

\begin{abstract}
Given a very general abelian fivefold $A$ and a principal polarization $\Theta \subset A$, we construct surfaces generating the algebraic part of the middle cohomology $H^{4}(\Theta, \mathbb{Q})$, and determine the intersection pairing between these surfaces. In particular, we obtain a new proof of the Hodge conjecture for $H^{4}(\Theta, \mathbb{Q})$ and show that it contains a copy of the root lattice of $E_{6}$.
\end{abstract}

\section{Contents}

Introduction 1

Notation 3

1. Prym varieties and Prym-embeddings 4

2. 27 surfaces 2

3. 27 curves 9

4. The one-parameter family of abelian fivefolds 10

5. Families of surfaces 12

6. A nodal fiber 14

$\begin{array}{ll}\text { 7. Proof of the main theorem } & 20\end{array}$

8. Appendix 21

References 28

\section{INTRODUCTION}

Let $A$ be a principally polarized abelian variety (ppav) of dimension $g \geq 4$ with smooth symmetric theta divisor $\Theta$. By the Lefschetz hyperplane theorem and Poincaré Duality (see, e.g., [IW15]), the cohomology of $\Theta$ is determined by that of $A$ except in the middle dimension $g-1$. The primitive cohomology of $\Theta$, in the sense of Lefschetz, is

$$
H_{\mathrm{pr}}^{g-1}(\Theta, \mathbb{Z}):=\operatorname{Ker}\left(H^{g-1}(\Theta, \mathbb{Z}) \stackrel{\cup[\Theta]}{\longrightarrow} H^{g+1}(\Theta, \mathbb{Z})\right)
$$


The primal cohomology of $\Theta$ is defined as (see [IW15] and [ITW17])

$$
\mathbb{K}:=\operatorname{Ker}\left(H^{g-1}(\Theta, \mathbb{Z}) \stackrel{i_{*}}{\rightarrow} H^{g+1}(A, \mathbb{Z})\right)
$$

where $i: \Theta \hookrightarrow A$ is the inclusion. This is a Hodge substructure of $H_{\mathrm{pr}}^{g-1}(\Theta, \mathbb{Z})$ of $\operatorname{rank} g !-\frac{1}{g+1}\left(\begin{array}{c}2 g \\ g\end{array}\right)$ and level $g-3$ while the primitive cohomology $H_{\mathrm{pr}}^{g-1}(\Theta, \mathbb{Z})$ has full level $g-1$.

The action of -1 splits $\mathbb{K}_{\mathbb{Q}}$ into the direct sum of its invariant piece $\mathbb{K}_{\mathbb{Q}}^{+}$and its anti-invariant piece $\mathbb{K}_{\mathbb{Q}}^{-}$. As shown in [IW18, Lemma 6.1], the Hodge structure $\mathbb{K}^{(-1)^{g}}$ has level $g-3$ while the Hodge structure $\mathbb{K}^{(-1)^{g-1}}$ has level $g-5$.

The primal cohomology $\mathbb{K}$ and its Hodge substructure $\mathbb{K}^{(-1)^{g-1}}$ are therefore interesting test cases for the general Hodge conjecture. The general Hodge conjecture predicts that $\mathbb{K}_{\mathbb{Q}}:=\mathbb{K} \otimes \mathbb{Q}$ is contained in the image, via Gysin pushforward, of the cohomology of a smooth (possibly reducible) variety of pure dimension $g-3$ (see [IW15]). This conjecture was proved in [IS95] and [ITW17] in the cases $g=4$ and $g=5$. When $g=4$, it also follows from the proof of the Hodge conjecture in [IS95] that, for $(A, \Theta)$ generic, $\mathbb{K}$ is a simple Hodge structure (isogenous to the third cohomology of a smooth cubic threefold). In this case the primal cohomology is fixed under the action of -1 .

In the case $g=5, \mathbb{K}_{\mathbb{Q}}^{+}$and $\mathbb{K}_{\mathbb{Q}}^{-}$have respective dimensions 6 and 72 . The space $\mathbb{K}_{\mathbb{Q}}^{+}$consists of Hodge classes ([IW18, Corollary 6.2]) while $\mathbb{K}_{\mathbb{Q}}^{-}$is simple [IW18]. It follows from the main result of [ITW17] and the Lefschetz $(1,1)$ theorem that the classes belonging to $\mathbb{K}_{\mathbb{Q}}^{+}$are algebraic. Here we describe explicit natural surfaces in $\Theta$ which represent these classes. Our main result is the following

Theorem 1. Suppose $(A, \Theta)$ is a general ppav of dimension 5.

(a) There are 27 smooth surfaces $V_{i} \subset \Theta$ (to be described below, up to translation), whose classes in $H^{4}(\Theta, \mathbb{Q})$ span $\mathbb{Q}[\Theta]^{2}+\mathbb{K}_{\mathbb{Q}}^{+}$(which is the space of Hodge classes if $A$ is very general).

(b) The sublattice of $\mathbb{K}^{+}$spanned by classes of the form $\left[V_{i}\right]-\left[V_{j}\right]$ is isometric to $H_{\mathrm{pr}}^{2}(X, \mathbb{Z})(-2)$ for any smooth cubic surface $X \subset \mathbb{P}^{3}$.

(c) There is a (non-canonical) bijection between the $V_{i}$ and the lines $L_{i}$ in $X$ such that the isometry sends $\left[V_{i}\right]-\left[V_{j}\right]$ to $\left[L_{i}\right]-\left[L_{j}\right]$.

We construct the surfaces in two different ways, both of which rely on the theory of Prym varieties. One uses Brill-Noether theory for Prym varieties [Wel85]; the other exhibits the surfaces as special subvarieties of $A$, in the sense of [Bea82]. By comparing these two constructions, exploiting a connection with the 27 lines on a cubic surface, we produce relations between the intersection numbers $\left[V_{i}\right] .\left[V_{j}\right]$. With these in hand, it remains to compute $\left[V_{i}\right]^{2}$ for all $i$. 
To do so, we adapt the one parameter degeneration of [IW18], whose central fiber is a compactification $\left(A_{0}, \Theta_{0}\right)$ of a semiabelian extension of a ppav $(B, \Xi)$ of dimension 4 . The limit theta divisor $\Theta_{0}$ is singular and birational to $B$.

Each limit surface $V_{0} \subset \Theta_{0}$ is birational to $\Xi_{\alpha} \cap \Xi_{\beta}$ for some $\alpha, \beta \in B$ (subscripts denote translation). We identify $V_{0}$ using a Hilbert polynomial calculation, and make sense of $\left[V_{0}\right]^{2}$ using the smoothness of the total space of theta divisors. Finally, we compute the degree of $\left[V_{0}\right]^{2}$ using properties of the Prym-embedded curves in $\Xi_{\alpha} \cap \Xi_{\beta}$, which were studied in [Iza95] and [Krä15].

The paper is organized as follows. In Section 1 we gather some known facts about Prym Varieties and prove some preliminary results about some subvarieties of Prym varieties that we will need later. In Section 2 we define the 27 surfaces and compute their intersection numbers with the exception of their self-intersection numbers. Section 3 contains analogous results for sets of 27 curve classes in abelian fourfolds that we will need later for our degeneration argument. In Section 4 we describe the one-parameter degeneration of abelian fivefolds and their theta divisors that we will use to prove our main result. In Section 5 we describe the one-parameter degenerations of surfaces that we use to compute the self-intersection numbers of the surfaces $V_{i}$, and in Section 6 we describe the central fibers of these families. We complete the proof of our main result in Section 7 by computing the self-intersection numbers of $V_{i}$, using the one-parameter families of surfaces. Finally, the Appendix, Section 8, contains some computations used in the paper. It also contains the computation of the ranks and Hodge numbers of $\mathbb{K}^{+}$and $\mathbb{K}^{-}$in all dimensions.

\section{NotATION}

If $C$ is a curve, then $\widetilde{C}$ always denotes a fixed étale double cover of $C$, with covering involution $\sigma: \widetilde{C} \rightarrow \widetilde{C}$ and quotient map $\pi: \widetilde{C} \rightarrow C$. For $c \in \widetilde{C}$ we denote $\sigma(c)$ by $c^{\prime}$. We denote by $C^{(d)}$ the $d^{\text {th }}$ symmetric power of $C$, and $G_{d}^{r}(C)$ (resp. $W_{d}^{r}(C)$ ) the space of linear systems (resp. complete linear systems) of degree $d$ and dimension $r$ (resp. at least $r$ ) on $C$. We typically denote a fixed element of $G_{d}^{r}(C)$ by $g_{d}^{r}$. The dual projective space of $g_{d}^{r}$ is denoted by $g_{d}^{r \vee}$. The notation $C_{1} \triangleq C_{2}$ means $C_{1}$ and $C_{2}$ are tetragonally related (see Paragraph 1.6).

As usual, $\mathcal{A}_{g}$ is the (coarse) moduli space of dimension $g$ principally polarized abelian varieties (ppav), $\mathcal{R}_{g}$ is the (coarse) moduli space of étale couble covers $\widetilde{C} \rightarrow C$ with $C$ of genus $g, \mathcal{M}_{g}$ is the (coarse) moduli space of smooth curves of genus $g$, and $\overline{\mathcal{M}}_{g}$ is its compactification parametrizing stable curves.

If $X$ is a scheme, then $\mathrm{CH}_{d}(X)$ is the Chow group of algebraic cycles on $X$ modulo rational equivalence.

If $L$ is a lattice then $L(n)$ is the lattice obtained by multiplying the intersection form by $n \in \mathbb{Z}$. 


\section{Prym varieties and Prym-embeddings}

1.1. Prym varieties. The Prym map $\mathcal{P}_{0}: \mathcal{R}_{g+1} \rightarrow \mathcal{A}_{g}$ sends a Beauville admissible cover $\widetilde{X} \stackrel{\pi}{\rightarrow} X$ (see [Bea77a]) to its Prym variety $\mathcal{P}_{0}(X):=\operatorname{Im}\left(\sigma^{*}-\mathrm{id}\right) \subset \operatorname{Pic}^{0}(\widetilde{X})$, where $\sigma: \widetilde{X} \rightarrow \widetilde{X}$ is the covering involution. The map $\mathcal{P}_{0}$ is surjective for $g \leq 5$, hence generically finite for $g=5$ by a dimension count. Its degree is 27 in this case [DS81].

1.2. Prym torsors. The Prym variety $\mathcal{P}_{0}(X)$ can also be defined as the identity component of $\operatorname{Ker}(\mathrm{Nm})$, where $\mathrm{Nm}: \operatorname{Pic}(\widetilde{X}) \rightarrow \operatorname{Pic}(X)$ is the norm map (which agrees with the push-forward on Chow groups $\left.\mathrm{CH}_{0}(\widetilde{X}) \stackrel{\pi_{*}}{\longrightarrow} \mathrm{CH}_{0}(X)\right)$. The kernel of $\mathrm{Nm}$ has a second component, a translate of $\mathcal{P}_{0}(X)$, which we denote by $\mathcal{P}_{0}^{-}(X)$. We will also use the fiber of Nm over $\omega_{X}$, whose components $\mathcal{P}_{2 g}(X)$ and $\mathcal{P}_{2 g}^{-}(X)$ consist of line bundles $L$ such that $h^{0}(L)$ is even or odd respectively.

1.3. Prym-embeddings. If $X$ is not hyperelliptic, then neither is $\tilde{X}$, as the push-forward $\pi_{*}$ : $\widetilde{X}^{(2)} \rightarrow X^{(2)}$ preserves linear equivalence. In this case $\iota(p):=\mathcal{O}_{\widetilde{X}}\left(p-p^{\prime}\right)$ defines an embedding $\widetilde{X} \hookrightarrow \mathcal{P}_{0}^{-}(X)$ (recall that $p^{\prime}:=\sigma(p)$ ). If $Z \subseteq \operatorname{Pic}(\widetilde{X})$, then translates of $\iota(\widetilde{X})$ contained in $Z$ are called Prym-embeddings of $\widetilde{X}$ in $Z$.

We also have the canonical morphism

$$
\begin{aligned}
& \widetilde{X}^{(2)} \longrightarrow \mathcal{P}_{0}(X) \\
& p+q \longmapsto[p, q]:=\iota(p)+\iota(q)=\mathcal{O}_{\tilde{X}}\left(p+q-p^{\prime}-q^{\prime}\right) .
\end{aligned}
$$

Note that $[p, q]=\kappa(p)-\kappa\left(q^{\prime}\right)$ for any Prym-embedding $\kappa: \widetilde{X} \hookrightarrow \mathcal{P}_{0}(X)$ (in fact any translate of $\iota$ ).

1.4. Brill-Noether loci. Given $r>0$, let $\mathcal{P}_{2 g}^{r}(X)$ be the locus in $\mathcal{P}_{2 g}(X) \amalg \mathcal{P}_{2 g}^{-}(X)$ where $h^{0}>r$ and $h^{0} \not \equiv r(\bmod 2)$. It inherits a scheme structure from the classical Brill-Noether locus $W_{2 g}^{r}(\widetilde{X}) \subseteq$ $\operatorname{Pic}(\widetilde{X})$, and is smooth at all $L$ with $h^{0}(L)=r+1$ if $X \in \mathcal{M}_{g+1}$ is sufficiently general [Wel85, Propsition 1.9 and Theorem 1.11].

The effective locus $\Theta_{2 g}=\Theta_{2 g}(X):=\mathcal{P}_{2 g}^{1}(X) \subset \mathcal{P}_{2 g}(X)$ defines a theta divisor in the sense that, for each $L_{0} \in \mathcal{P}_{2 g}(X)$, the translate $\Theta_{2 g}-L_{0}$ is a theta divisor for $\mathcal{P}_{0}(X)$. Choosing $L_{0}$ determines a group structure on $\mathcal{P}_{2 g}(X)$, where inversion is given by $L \mapsto L_{0}^{2}-L$. If $L_{0}$ is a theta characteristic (meaning $L_{0}^{2} \cong \omega_{\tilde{X}}$ ), this morphism (the residuation map) preserves $h^{0}(L)$ by Serre duality. In this case $\Theta_{2 g}-L_{0}$ is a symmetric theta divisor.

By [Iza95, Proposition 3.11], the second Brill-Noether locus $\widetilde{X}_{\lambda}:=\mathcal{P}_{2 g}^{2}(X) \subseteq \mathcal{P}_{2 g}^{-}(X)$ consists of those $L$ for which $\iota(\widetilde{X})+L \subset \Theta_{2 g}$. Each $p \in \widetilde{X}$ defines an embedding

$$
W_{p}:=\widetilde{X}_{\lambda}+\iota(p)=\left\{L \in \mathcal{P}_{2 g}(X) \mid h^{0}(L(-p))>1\right\} \subset \Theta_{2 g} .
$$

When $g=5$, these are the surfaces we will use to generate $\mathbb{K}^{+}$. In order to understand them better, we will show that the $W_{p}$ are special subvarieties in the sense of Beauville [Bea82]. 
1.5. Special subvarieties. Given a $g_{d}^{r}$ on $X$ with $2 r<d \leq 2 g$, the associated special subvarieties of the symmetric power $\widetilde{X}^{(d)}$ are the connected components $S_{i}$ of the fiber product

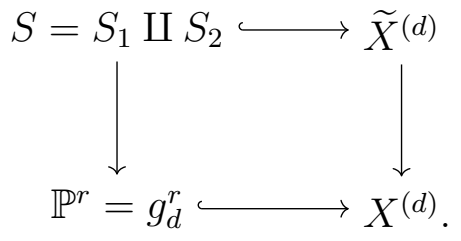

If the base locus of the $g_{d}^{r}$ is reduced, then so is $S$. When $d \leq r+g$, we also consider the special subvarieties $T_{i} \subseteq \widetilde{X}^{(2 g-d)}$ associated to the residual linear system $g_{2 g-d}^{r+g-d}$. After choosing the indices appropriately, for each $D \in T_{i}$, the image of $S_{i}+D \subseteq \widetilde{X}^{(2 g)}$ in $\operatorname{Pic}^{2 g}(\widetilde{X})$ is a subvariety $V_{D} \subseteq \Theta_{2 g}$. On the other hand $S_{3-i}+D$ maps into $\mathcal{P}_{2 g}^{-}(X)$. The $V_{D}$ are called special subvarieties of $\Theta_{2 g}$.

1.6. The tetragonal construction. For a (base point free) $g_{4}^{1}$ the above is known as the tetragonal construction [Don92]. Each special subvariety $\widetilde{X}_{i}:=S_{i}$ is a smooth curve, assuming the fibers of $X \rightarrow g_{4}^{1 \vee} \cong \mathbb{P}^{1}$ have at most one ramification point, with index at most 3 . The quotients $\widetilde{X}_{i} \rightarrow X_{i}$ induced by the covering involution $\widetilde{X}^{(4)} \stackrel{\sigma_{*}}{\longrightarrow} \widetilde{X}^{(4)}$ have the same Prym variety as $\widetilde{X} \rightarrow X$. Each $X_{i}$ carries a $g_{4}^{1}$ for which the associated special subvarieties of $\widetilde{X}_{i}^{(4)}$ are $\widetilde{X}$ and $\widetilde{X}_{3-i}$. We say that the $\tilde{X}_{i} \rightarrow X_{i}$ are tetragonally related to $\widetilde{X} \rightarrow X$, written $X_{i} \triangleq X$, or that $\left(X, X_{1}, X_{2}\right)$ is a tetragonal triple.

If $X \triangleq Y$, given a Prym-embedding $\kappa: \widetilde{X} \hookrightarrow \mathcal{P}_{0}(X)$ and a lift $D \in \widetilde{Y}^{(2 g-4)}$ of a divisor in the $g_{2 g-4}^{g-3}$ on $Y$, there is a line bundle $L \in \mathcal{P}_{2 g}(Y)$ and an isomorphism $\varphi: \mathcal{P}_{0}(X) \rightarrow \mathcal{P}_{0}(Y)$ such that $\varphi(\kappa(p))=L^{-1}(P+D)$ for all $p \in \widetilde{X}$ corresponding to $P \in \widetilde{Y}^{(4)}$. In particular $\varphi(\kappa(\widetilde{X}))=V_{D}-L$. Moreover, if $P, Q^{\prime} \in \widetilde{Y}^{(4)}$ correspond to $p, q^{\prime} \in \widetilde{X}$, then

$$
\varphi([p, q])=L^{-1}(P+D) \otimes L\left(-Q^{\prime}-D\right)=\mathcal{O}_{\widetilde{Y}}\left(P-Q^{\prime}\right)
$$

1.7. Prym-curves. A Prym-curve for an abelian variety $A$ is an admissible cover $\widetilde{X} \stackrel{\pi}{\rightarrow} X$ such that $\mathcal{P}_{0}(X) \cong A$. By abuse of notation, we will often denote a Prym-curve by its base curve $X$, the double cover $\widetilde{X} \stackrel{\pi}{\rightarrow} X$ being implicit.

Definition 1.1. We call a Prym-curve $\widetilde{X} \stackrel{\pi}{\rightarrow} X \operatorname{good}$ if $X$ is smooth (hence $\pi$ is étale) and $X$ is not hyperelliptic, trigonal or bielliptic.

When $A$ is general and $g \geq 5$, every Prym-curve for $A$ is good (see [Mum74, §7], [Rec74] and $[$ Don92, §3]). When $A$ is general and $g=4$, the fiber of the Prym map at $A$ always contains singular Prym curves. However, every smooth Prym-curve is good.

Lemma 1.2. Let $X$ be a good Prym-curve and set $\Theta:=\Theta_{2 g}(X)$. If $p, q, r \in \widetilde{X}$ are such that $p, p^{\prime}$, $q$ and $r$ are distinct, then: 
(a) $\Theta \cap \Theta_{[p, q]}=V_{p+q}$.

(b) $\Theta \cap \Theta_{[p, q]} \cap \Theta_{[p, r]}=W_{p} \cup V_{p+q+r}$.

(c) $\Theta_{[p, q]} \cap \Theta_{[p, r]} \cap \Theta_{[q, r]}=\left(W_{p}+[q, r]\right) \cup V_{p+q+r}$.

(d) $W_{p}$ and $W_{p}+[q, r]$ are algebraically equivalent in $\Theta_{[p, q]} \cap \Theta_{[p, r]}$.

If $s \in \tilde{X}$ is such that $\pi_{*}(p+q+r+s) \in X^{(4)}$ moves in a pencil, then

(e) $V_{p+q+r}=V_{p+q+r+s} \cup V_{p+q+r+s^{\prime}}$.

Proof. See the proof of [BD87, Proposition 1] for (a) and (b), and [Iza95, Proposition 2.4.1] for (e). Using (b),

$\Theta_{[p, q]} \cap \Theta_{[p, r]} \cap \Theta_{[q, r]}=\left(\Theta_{\left[p, r^{\prime}\right]} \cap \Theta_{\left[p, q^{\prime}\right]} \cap \Theta\right)+[q, r]=\left(W_{p} \cup V_{p+q^{\prime}+r^{\prime}}\right)+[q, r]=\left(W_{p}+[q, r]\right) \cup V_{p+q+r}$,

giving (c). The algebraic equivalence $\Theta \sim \Theta_{[q, r]}$ on $\mathcal{P}_{2 g}(X)$ restricts to

$$
\Theta \cap \Theta_{[p, q]} \cap \Theta_{[p, r]} \sim \Theta_{[q, r]} \cap \Theta_{[p, q]} \cap \Theta_{[p, r]}
$$

on $\Theta_{[p, q]} \cap \Theta_{[p, r]}$. Using (b) and (c), this gives (d).

Lemma 1.3. Suppose $X \triangleq Y$ are good Prym-curves for $(A, \Theta)$. If $p \in \widetilde{X}$ corresponds to $P \in \widetilde{Y}^{(4)}$, there is an isomorphism $\psi: \mathcal{P}_{2 g}(X) \rightarrow \mathcal{P}_{2 g}(Y)$ for which $\psi\left(W_{p}\right)=V_{P}$.

Proof. Let $L \in \mathcal{P}_{2 g}(Y)$ and $\varphi: \mathcal{P}_{0}(X) \rightarrow \mathcal{P}_{0}(Y)$ be as in Paragraph 1.6. Choose theta characteristics $L_{X}$ and $L_{Y}$ on $\widetilde{X}$ and $\widetilde{Y}$ respectively, and define $\psi$ by the formula

$$
\psi(M):=\varphi\left(M-L_{X}\right)+L_{Y}
$$

We may choose $L_{Y}$ so that $\psi$ maps $\Theta_{2 g}(X)$ onto $\Theta_{2 g}(Y)$. For $M \in V_{P}$ and $q \in \widetilde{X}$ (corresponding to $\left.Q \in \tilde{Y}^{(4)}\right)$,

$$
\varphi(\iota(q)-\iota(p))+M=\varphi\left[q, p^{\prime}\right]+M=M(Q-P) \in \Theta_{2 g}(Y) .
$$

(note that $M(-P)$ is effective). Applying $\psi^{-1}$ shows that

$$
\iota(q)-\iota(p)+\psi^{-1}(M) \in \Theta_{2 g}(X)
$$

for all $q \in \widetilde{X}$, which means $\psi^{-1}(M)-\iota(p) \in \widetilde{X}_{\lambda}$. Therefore $V_{P} \subseteq \psi\left(W_{p}\right)$.

As a special subvariety, $V_{P}$ has class $\frac{1}{3}[\Theta]^{3} \in H^{6}(A, \mathbb{Z})$ [Bea82, Théorème 1]. On the other hand $V_{p+q+r}$ has class $\frac{2}{3}[\Theta]^{3}$ whenever $q, r \in \widetilde{X}$. Hence, by Lemma $1.2(\mathrm{~b}), \psi_{*}\left[W_{p}\right]=\left[V_{P}\right]$. 
1.8. $\lambda$-classes. Let $X$ be a Prym-curve for $(A, \Theta)$. By definition, residuation sends $W_{p}$ to $W_{p^{\prime}}$ for all $p \in \widetilde{X}$. The algebraic equivalence class of $W_{p}$ in $\Theta_{2 g}$ is independent of $p \in \widetilde{X}$, and therefore fixed by residuation. Given an isomorphism $\psi: \mathcal{P}_{0}(X) \rightarrow A$, there is a unique theta characteristic $L_{0}$ on $\widetilde{X}$ such that $\psi\left(\Theta_{2 g}-L_{0}\right)=\Theta$. Let $\left[\widetilde{X}_{\lambda}\right]$ be the algebraic equivalence class of $\psi\left(W_{p}-L_{0}\right) \subset \Theta$; it is independent of $p \in \widetilde{X}$ and fixed by -1 . If $(A, \Theta)$ is very general, then $\operatorname{Aut}(A, \Theta)=\{ \pm 1\}$, which means $\left[\widetilde{X}_{\lambda}\right]$ does not depend on $\psi$.

Corollary 1.4. If $(X, Y, Z)$ is a tetragonal triple of good Prym-curves for a very general Prym variety $(A, \Theta)$, then $\left[\widetilde{X}_{\lambda}\right]+\left[\widetilde{Y}_{\lambda}\right]+\left[\widetilde{Z}_{\lambda}\right]=[\Theta]^{2}$.

Proof. If $p+q+r+s \in \widetilde{Y} \subset \widetilde{X}^{(4)}$ lifts a reduced divisor of the $g_{4}^{1}$ on $X$, and $\Xi:=\Theta_{2 g}(X)$, then

$$
\Xi \cap \Xi_{[p, q]} \cap \Xi_{[p, r]}=W_{p} \cup V_{p+q+r+s} \cup V_{p+q+r+s^{\prime}}
$$

by Lemma 1.2 parts (b) and (e). Choosing isomorphisms as needed, Lemma 1.3 implies that the classes of (the images of) $W_{p}, V_{p+q+r+s}$ and $V_{p+q+r+s^{\prime}}$ in $\Theta$ are $\left[\widetilde{X}_{\lambda}\right],\left[\widetilde{Y}_{\lambda}\right]$ and $\left[\widetilde{Z}_{\lambda}\right]$ respectively.

\section{27 SURFACES}

In this section $g=5$ and the $\widetilde{X}_{\lambda}$ are surfaces. The fiber of $\mathcal{P}_{0}$ at a general ppav $(A, \Theta) \in \mathcal{A}_{5}$ consists of 27 Prym-curves, and the tetragonal correspondence between them is isomorphic to the incidence correspondence for the lines on a smooth cubic surface [Don92, 4.2]. This is almost enough to compute the intersection pairing between the $\left[\widetilde{X}_{\lambda}\right]$.

2.1. 27 lines. The lines in the cubic surface obtained by blowing up 6 points $p_{1}, \ldots, p_{6} \in \mathbb{P}^{2}$ in general position (with respect to lines and conics) can be described as follows:

- The exceptional divisor $E_{i}$ over each point $p_{i}$.

- The proper transform $F_{i j}$ of the line joining $p_{i}$ to $p_{j}$, for $i<j$. If $i>j$ we set $F_{i j}:=F_{j i}$.

- The proper transform $G_{j}$ of the conic containing $p_{i}$ for $i \neq j$.

Two lines meet if and only if they both belong to a triple of the form $\left(E_{i}, F_{i j}, G_{j}\right)$ with $i \neq$ $j$, or $\left(F_{i j}, F_{k l}, F_{m n}\right)$ with $\{i, j, k, l, m, n\}=\{1, \ldots, 6\}$. The automorphism group $W\left(\mathrm{E}_{6}\right)$ of this configuration acts transitively on lines and sixers (i.e., sextuples of mutually skew lines) [Dol12, Proposition 9.1.4]. Any permutation of the indices acts on the sixer $\left(E_{1}, \ldots, E_{6}\right)$ and on the cubic surface.

Theorem 2.1. Let $(A, \Theta)$ be a very general ppav of dimension 5. Suppose that $\left[\widetilde{X}_{\lambda}\right]^{2}=16$ whenever $X$ is a Prym-curve for $(A, \Theta)$. If $X$ and $Y$ are non-isomorphic Prym-curves, then

$$
\left[\widetilde{X}_{\lambda}\right] \cdot\left[\widetilde{Y}_{\lambda}\right]= \begin{cases}12 & \text { if } X \triangleq Y \\ 14 & \text { otherwise }\end{cases}
$$


Proof. First, suppose that $X \triangleq Y$ and form the tetragonal triple $(X, Y, Z)$. Let $\langle-,-\rangle$ be the pairing on (formal sums of) Prym-curves induced by the intersection form on $\Theta$, so that

$$
\langle X, X+Y+Z\rangle=\left[\widetilde{X}_{\lambda}\right] \cdot\left(\left[\widetilde{X}_{\lambda}\right]+\left[\widetilde{Y}_{\lambda}\right]+\left[\widetilde{Z}_{\lambda}\right]\right)=\left[\widetilde{X}_{\lambda}\right] \cdot[\Theta]^{2}=\frac{g !}{3}=40
$$

by Corollary 1.4 and the fact that $\left[\tilde{X}_{\lambda}\right]=\frac{1}{3}[\Theta]^{3}$ in $A$ (see Lemma 1.3). Permuting $X, Y, Z$, we obtain

$$
\langle X, Y+Z\rangle=\langle Y, Z+X\rangle=\langle Z, X+Y\rangle=40-16=24
$$

so $\langle X, Y\rangle=\langle X, Z\rangle=\langle Y, Z\rangle=12$.

Now, suppose that $X \notin Y$. Choose a Prym-curve $Z_{1}$ tetragonally related to $X$ and $Y$. As above, we form triples $\left(X, Z_{1}, X_{1}\right)$ and $\left(Y, Z_{1}, Y_{1}\right)$ so that

$$
40=\left\langle X, Y+Z_{1}+Y_{1}\right\rangle=\left\langle X_{1}, Y+Z_{1}+Y_{1}\right\rangle=\left\langle Y, X+Z_{1}+X_{1}\right\rangle=\left\langle Y_{1}, X+Z_{1}+X_{1}\right\rangle
$$

and hence

$$
\langle X, Y\rangle=28-\left\langle X_{1}, Y\right\rangle=\left\langle X_{1}, Y_{1}\right\rangle=28-\left\langle X, Y_{1}\right\rangle
$$

This does not immediately give $\langle X, Y\rangle=14$, but we can find more equations of the form (2.1) by varying $X, Y$ and $Z_{1}$. For this, it helps to label the Prym-curves as in Paragraph 2.1. We have similar identities for all the 4-tuples of surfaces obtained, as above, from a pair of skew lines in the cubic surface. For distinct indices $i, j, k$, we have the following pairs of tetragonally related triples
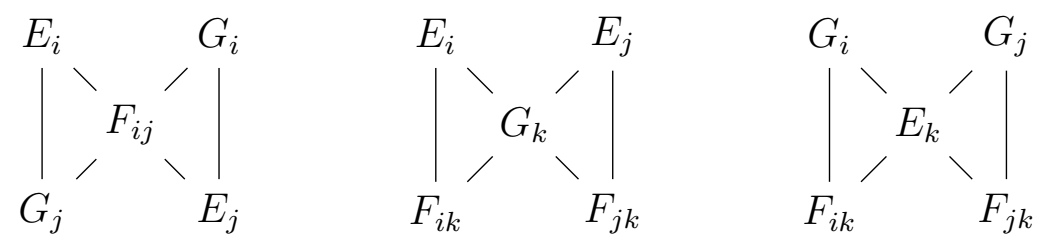

The application of (2.1) gives

$$
\begin{gathered}
\left\langle E_{i}, G_{i}\right\rangle=\left\langle G_{j}, E_{j}\right\rangle=28-\left\langle E_{i}, E_{j}\right\rangle=28-\left\langle G_{i}, G_{j}\right\rangle, \\
\left\langle E_{i}, E_{j}\right\rangle=\left\langle F_{i k}, F_{j k}\right\rangle=28-\left\langle E_{i}, F_{j k}\right\rangle=28-\left\langle E_{j}, F_{i k}\right\rangle, \\
\left\langle G_{i}, G_{j}\right\rangle=\left\langle F_{i k}, F_{j k}\right\rangle=28-\left\langle G_{i}, F_{j k}\right\rangle=28-\left\langle G_{j}, F_{i k}\right\rangle .
\end{gathered}
$$

It follows from the equations above that all intersection numbers of the form $\left\langle E_{i}, E_{j}\right\rangle,\left\langle G_{i}, G_{j}\right\rangle$ or $\left\langle F_{i k}, F_{j k}\right\rangle$ are equal and all intersection numbers of the form $\left\langle E_{i}, G_{i}\right\rangle,\left\langle E_{i}, F_{j k}\right\rangle$ or $\left\langle G_{i}, F_{j k}\right\rangle$ are equal. Now note that we also have the surfaces obtained from the tetragonally related triple $\left(F_{12}, F_{34}, F_{56}\right)$. Intersecting the sum of these three surfaces with $E_{1}$, we obtain:

$$
\left\langle E_{1}, F_{34}\right\rangle+\left\langle E_{1}, F_{56}\right\rangle=28
$$


Since $\left\langle E_{1}, F_{34}\right\rangle=\left\langle E_{1}, F_{56}\right\rangle$, we obtain

$$
\left\langle E_{1}, F_{34}\right\rangle=14
$$

and hence all the other intersection numbers above are also equal to 14 .

\section{27 CURVES}

For a general ppav $B$ of dimension $g=4$, the fiber of $\mathcal{P}_{0}$ over $B$ is two-dimensional, a double cover of the Fano surface of lines on a particular cubic threefold [Don92, 5.1], [Iza95, 6.27] . The covering involution is given by $X \mapsto X_{\lambda}$, where $\tilde{X}_{\lambda} \rightarrow X_{\lambda}$ is the quotient by residuation [Iza95, $3.11,3.13]$. Each point $\alpha \in B$ determines a hyperplane section of the cubic threefold, and a choice of Prym-curve $X$ lying over each of its lines [Iza95, 3.16, 4.8, 4.9, 5.7, 5.10]. The curves $\widetilde{X}_{\lambda}$ admit embeddings in $\Xi \cap \Xi_{\alpha}$, where $\Xi$ is a theta divisor for $B$. In this section we prove an analog of Theorem 2.1 for these embeddings.

For $0 \neq \alpha \in B$, one has $W_{p} \subset \Theta \cap \Theta_{\alpha}$ if and only if $\alpha=[p, q]$ for some $q \in \widetilde{X}$ [Iza95, 3.16].

Definition 3.1. An $\alpha$-curve is a Prym-curve $X$ for $B$ such that $W_{p} \subset \Theta \cap \Theta_{\alpha}$ for some $p \in \widetilde{X}$, where $\Theta:=\Theta_{8}(X)$ is the canonical translate of $\Xi$ in $\mathcal{P}_{8}(X)$.

Lemma 3.2. If $X$ is an $\alpha$-curve such that $X$ and $X_{\lambda}$ are good, then the translates of $\widetilde{X}_{\lambda}$ in $\Xi \cap \Xi_{\alpha}$ are algebraically equivalent.

Proof. Pick $q, r \in \widetilde{X}$ such that $\alpha=\left[q^{\prime}, r\right]$. The two embeddings of $\widetilde{X}_{\lambda}$ in $\Theta \cap \Theta_{\alpha}$ are $W_{q^{\prime}}$ and $W_{r}$. Given $p \in \widetilde{X} \backslash\left\{q, q^{\prime}, r, r^{\prime}\right\}$, it follows that the embeddings of $\widetilde{X}_{\lambda}$ in $\left(\Theta \cap \Theta_{\alpha}\right)+[p, q]=\Theta_{[p, q]} \cap \Theta_{[p, r]}$ are $W_{q^{\prime}}+[p, q]=W_{p}$ and $W_{r}+[p, q]=W_{p}+[q, r]$, which are algebraically equivalent by Lemma $1.2(\mathrm{~d})$. Now translate back to $\Xi \cap \Xi_{\alpha}$.

3.1. Curve classes. As a consequence there is a well-defined class $\left[\widetilde{X}_{\lambda}\right] \in H^{2}\left(\Xi \cap \Xi_{\alpha}, \mathbb{Z}\right)$. It is invariant under the involution $\beta \mapsto \alpha-\beta$ on $\Xi \cap \Xi_{\alpha}$. Since $\gamma_{X}:=\left[\widetilde{X}_{\lambda}\right]-\frac{1}{3}[\Xi] \in H^{2}\left(\Xi \cap \Xi_{\alpha}, \mathbb{Q}\right)$ pushes forward to 0 in $B$, it belongs to $\mathbb{W}_{\mathbb{Q}}^{+}$, where $\mathbb{W} \subset H^{2}\left(\Xi \cap \Xi_{\alpha}, \mathbb{Z}\right)$ is the primal cohomology for $\Xi \cap \Xi_{\alpha} \hookrightarrow B$. Krämer showed that $\mathbb{W}^{+} \cong \mathrm{E}_{6}(-2)\left[\right.$ Krä15, 5.1], and that $\gamma_{X}$ is a norm-minimizing element of the dual lattice [Krä15, Lemma 7.2]. We refine his calculation using the argument of Theorem 2.1, after establishing the following Lemma.

Lemma 3.3. Suppose $B$ is very general and $\alpha \in B$ is general.

(a) If $X$ is an $\alpha$-curve, then $X$ and $X_{\lambda}$ are good.

(b) For $\alpha$-curves $X \triangleq Y$, the other curve in the tetragonal triple $(X, Y, Z)$ is also an $\alpha$-curve, and $\left[\widetilde{X}_{\lambda}\right]+\left[\widetilde{Y}_{\lambda}\right]+\left[\widetilde{Z}_{\lambda}\right]=[\Xi]$ in $\Xi \cap \Xi_{\alpha}$. 
Proof. Since $B$ is general, the fiber of $\mathcal{P}_{0}$ over $B$ contains a dense open subset of good Prym-curves [Iza95, 3.3, 3.10]. The complement $Z$ is therefore (at most) one-dimensional. Recall that $X$ is an $\alpha$-curve if and only if $\alpha$ belongs to the surface $\Sigma(X):=\{[p, q] \mid p, q \in \widetilde{X}\}$ [Iza95, 3.16]. For dimension reasons, we may assume that $\alpha \notin \cup_{X \in Z \cup \lambda(Z)} \Sigma(X)$, which gives (a).

For any $\alpha \in B$ and Prym-curves $X \triangleq Y$ such that $\alpha \in \Sigma(X) \cap \Sigma(Y)$, call $\alpha$ good for $X{ }^{\diamond} Y$ if there is a reduced divisor $p+q+r+s \in \widetilde{Y}$ such that $\alpha=[p, q]$. According to [Iza95, 5.9], $\Sigma(X) \cap \Sigma(Y)$ has (possibly impure) dimension one, and a general point on it is good for $X \triangleq Y$. Hence the set of elements of $\Sigma(X)$ which are not good for $X \triangleq Y$ for some $Y$ is of dimension $\leq 1$. The union of these sets for all Prym-curves $X$ is at most a threefold in $B$. So a general $\alpha \in B$ is good for all pairs $X \triangleq Y$ such that $\alpha \in \Sigma(X) \cap \Sigma(Y)$.

Given $\alpha$-curves $X \triangleq Y$, we may pick $p+q+r+s \in \tilde{Y}$ as above, and form the tetragonal triple $(X, Y, Z)$. The argument of Corollary 1.4 completes the proof of (b).

Proposition 3.4. Suppose $\alpha \in B$ is general. If $X$ and $Y$ are $\alpha$-curves, then

$$
\left[\widetilde{X}_{\lambda}\right] \cdot\left[\widetilde{Y}_{\lambda}\right]= \begin{cases}0 & \text { if } X=Y \\ 4 & \text { if } X \triangleq Y \\ 2 & \text { otherwise }\end{cases}
$$

Proof. Since $\alpha$ is general, $\Xi \cap \Xi_{\alpha}$ is smooth, so the adjunction formula shows that $\left[\widetilde{X}_{\lambda}\right] \cdot\left[\widetilde{X}_{\lambda}\right]=0$.

For the other cases, suppose that $B$ is very general. The tetragonal correspondence between $\alpha$-curves is isomorphic to the incidence correspondence between the 27 lines on a smooth cubic surface [Iza95, 5.9, 5.12, 6.8]. We may therefore argue as in Theorem 2.1 using Lemma 3.3.

Since intersection numbers are constant in smooth families, the result holds even when $(B, \Xi)$ has extra automorphisms (but remains sufficiently general).

Corollary 3.5. If $\alpha \in B$ is general, then the invariant primal cohomology $\mathbb{W}^{+} \subset H^{2}\left(\Xi \cap \Xi_{\alpha}, \mathbb{Z}\right)$ is freely generated by the classes $\delta_{i}:=\left[\widetilde{X}_{i \lambda}\right]-\left[\widetilde{Y}_{\lambda}\right]$, for any collection of $\alpha$-curves $X_{1}, \ldots, X_{6}, Y$ such that the $X_{i}$ are mutually tetragonally unrelated and $Y$ is related to exactly two of the $X_{i}$.

Proof. If $X$ and $Y$ are $\alpha$-curves corresponding to lines $E$ and $F$ on a cubic surface, then Proposition 3.4 says that $\left[\widetilde{X}_{\lambda}\right] \cdot\left[\widetilde{Y}_{\lambda}\right]=2(E . F+1)$. Thus, the matrix with entries $\delta_{i} \cdot \delta_{j}$ is a Gram matrix for $\mathrm{E}_{6}(-2)$. In particular, its determinant is the discriminant of $\mathbb{W}^{+}$, namely 192 . The map $\mathbb{Z}^{6} \rightarrow \mathbb{W}^{+}$ defined by the $\delta_{i}$ has to be invertible for this to hold.

\section{The one-Parameter Family of ABELIAN FIVEFoldS}

We summarize the construction of the family of theta divisors from [IW18]. The goal is to produce a rank one degeneration with smooth total space. 
4.1. Enriques and K3 surfaces. Let $R$ be a very general Enriques surface and $\rho: \widetilde{R} \rightarrow R$ the K3 étale double cover corresponding to the canonical class (which is 2-torsion) $K_{R} \in \operatorname{Pic}(R)$. Let $H$ be a very ample line bundle on $R$ with $H^{2}=10$. A general element in the linear system $|H| \cong \mathbb{P}^{5}$ is a smooth curve of genus 6; such curves are parameterized by the Zariski open subset $|H| \backslash D$, where $D$ is the dual variety of the embedding of $R$ in $|H|^{\vee}$. For each curve $C \in|H| \backslash D$, we obtain a nontrivial étale double cover $\widetilde{C}:=\rho^{-1}(C) \rightarrow C$. Associating to such a cover its Prym variety $\mathcal{P}_{0}(C)$ defines a morphism from $|H| \backslash D$ to $\mathcal{A}_{5}$ :

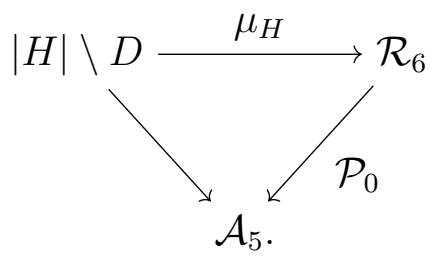

The linear systems $|H|$ form a projective bundle $\mathcal{H}$ over the moduli space of Enriques surfaces, and the maps $\mu_{H}$ are restrictions of a rational map $\mu: \mathcal{H} \rightarrow \mathcal{R}_{6}$. Mori and Mukai [MM83] showed that $\mu$ is dominant.

4.2. A family of curves. Suppose $T \cong \mathbb{P}^{1} \subset|H|$ is a Lefschetz pencil, i.e., it is transverse to the dual variety $D$. Then the singular curves of the pencil consist of finitely many irreducible nodal curves. Denote by $\mathcal{C}:=\mathrm{Bl}_{10} R$ (resp. $\widetilde{\mathcal{C}}:=\mathrm{Bl}_{20} \widetilde{R}$ ) the blow-up of $R$ (resp. $\widetilde{R}$ ) along the base locus of $T$ (resp. $\rho^{*} T$ ). We obtain a family of irreducible étale double covers parameterized by $T$ :

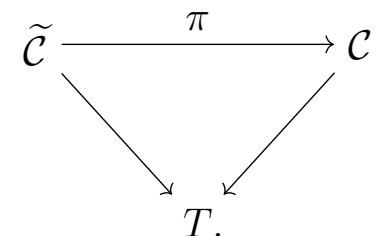

4.3. Singular fibers. There are 42 points $t_{1}, \ldots, t_{42} \in T$ where the fiber $C_{i}:=\mathcal{C}_{t_{i}}$ of the family $\mathcal{C} \rightarrow T$ is singular. The Prym varieties of the double covers in this family are well-defined as semiabelian varieties. They are abelian at the smooth fibers and have a rank 1 toric part at the singular fibers. This family of Prym varieties and their theta divisors can be compactified to families

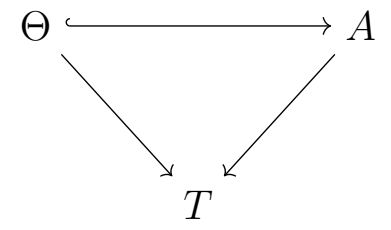

with smooth total spaces. The fibers $\Theta_{i} \subset A_{i}$ over $t_{i}$ have the following descriptions.

Denote by $\left(B_{i}, \Xi_{i}\right)$ the Prym variety of $\widetilde{X}_{i} \rightarrow X_{i}$, where $X_{i}$ (resp. $\widetilde{X}_{i}$ ) is the normalization of $C_{i}$ (resp. $\widetilde{C}_{i}$ ). It is a ppav of dimension 4. The semi-abelian Prym variety of $\widetilde{C}_{i} \rightarrow C_{i}$ is an extension of $B_{i}$ by $\mathbb{C}^{\times}$. Such an extension is determined by a point $\beta \in B_{i}$, well-defined up to \pm 1 . The 
normalization $P_{i}$ of $A_{i}$ is the $\mathbb{P}^{1}$-bundle over $B_{i}$ associated to this semi-abelian variety, and $A_{i}$ is obtained from $P_{i}$ by identifying the $\infty$-section $B_{\infty} \cong B_{i}$ with the 0 -section $B_{0} \cong B_{i}$ after translation by $\beta$. The normalization of $\Theta_{i}$ is the blow-up $\widetilde{B}_{i} \rightarrow B_{i}$ along $\Xi_{i} \cap \Xi_{i \beta}$. To recover $\Theta_{i}$ from $\widetilde{B}_{i}$, one glues the proper transforms of $\Xi_{i}$ and $\Xi_{i \beta}$ after translation by $\beta$.

4.4. Correcting the degree. In order to construct surfaces in a fiber $\Theta_{t}$, it it more natural to work in degree 10, i.e., we should have $A_{t}=\mathcal{P}_{10}\left(\mathcal{C}_{t}\right)$ and $\Theta_{t}=\Theta\left(\mathcal{C}_{t}\right)$. The construction of these families is similar to the degree zero case $[\mathrm{AFS} 15, \S 3.5]$. To check that their total spaces remain smooth, one can use étale-local (or local analytic) sections of $\Theta \rightarrow T$ to show that the families in degree 0 and 10 are étale-locally (or locally analytically) isomorphic. Alternatively, if one is willing to throw away the smooth fibers $A_{t}$ for which $\Theta_{t}$ is singular (which is harmless for our purposes), it is easy to prove directly (as in $[\mathrm{IW} 18,2.5]$ ) that $\Theta$ is nonsingular.

\section{FAMILIES OF SURFACES}

We construct families of special surfaces in the fibers of $\Theta \rightarrow T$.

5.1. Nets of degree 6 . Suppose $C \in \overline{\mathcal{M}}_{6}$ is irreducible and has at most one node. Given a $g_{6}^{2}$ on $C$, i.e., a net of degree 6 , and an étale double cover $\widetilde{C} \rightarrow C$, one can define special surfaces $S \subset \widetilde{C}^{(6)}$ as in the introduction. The relative version of this does not work over $T$ because the fibers of $\mathcal{C} \rightarrow T$ have no canonical choice of $g_{6}^{2}$. We will fix this by passing to a new base $U$; the map $U \rightarrow T$ should be unramified in order to preserve the smoothness of $\Theta$. There is a non-empty Zariski open subset $\mathcal{U}_{6}^{2} \subset \overline{\mathcal{M}}_{6}$ parameterizing curves $C$ with $\left|G_{6}^{2}(C)\right|=5$. Let $\Delta_{0} \subset \overline{\mathcal{M}}_{6}$ be the boundary component whose generic points parameterize irreducible curves.

Lemma 5.1. The intersection $\mathcal{U}_{6}^{2} \cap \Delta_{0}$ is not empty.

Proof. Suppose $C \in \Delta_{0}$ is general and let $X \stackrel{\nu}{\rightarrow} C$ be its normalization. Let $p, q \in X$ be the points over the node $r \in C$. Recall that $\operatorname{Pic}^{6}(C) \stackrel{\nu^{*}}{\longrightarrow} \operatorname{Pic}^{6}(X)$ is a $\mathbb{C}^{\times}$-torsor with fiber

$$
\mathbb{P}\left(\left.\left.L\right|_{p} \oplus L\right|_{q}\right) \backslash\left\{\left.L\right|_{p} \oplus 0,\left.0 \oplus L\right|_{q}\right\}
$$

over $L \in \operatorname{Pic}^{6}(X)$ [OS79, Corollary 12.4]. Given $L^{\prime} \in \operatorname{Pic}^{6}(C)$ with $\nu^{*} L^{\prime} \cong L$, the corresponding line in $\left.\left.L\right|_{p} \oplus L\right|_{q}$ is the kernel of the subtraction map $\left.\left.\left.L\right|_{p} \oplus L\right|_{q} \rightarrow L^{\prime}\right|_{r}$. This map is the evaluation at the node of the surjection in the following exact sequence, obtained by tensoring $\mathcal{O}_{C} \rightarrow \nu_{*} \mathcal{O}_{X}$ with $L^{\prime}$ :

$$
\left.0 \rightarrow L^{\prime} \rightarrow \nu_{*} L \rightarrow L^{\prime}\right|_{r} \rightarrow 0
$$


If $h^{0}\left(L^{\prime}\right)=3$ then $(5.1)$ forces $h^{0}(L)=3$ by Clifford's theorem and the genericity of $X$. Thus we may identify the maps $\left.H^{0}(X, L) \rightarrow L\right|_{w}$ and $\left.H^{0}\left(C, L^{\prime}\right) \rightarrow L^{\prime}\right|_{\nu(w)}$ for each $w \in X$. In particular

$$
H^{0}(X, L(-p-q))=H^{0}(X, L(-p))=H^{0}(X, L(-q))
$$

is two-dimensional (as $X$ is neither hyperelliptic nor trigonal, it has no $g_{5}^{2}$ ).

Conversely, if $h^{0}(L(-p-q))=h^{0}(L)-1=2$, then exactly one $L^{\prime}$ lying over $L$ satisfies $h^{0}\left(L^{\prime}\right)=3$. Indeed, if $h^{0}\left(L^{\prime}\right)=h^{0}(L)$ then the surjection in (5.1) vanishes on global sections, i.e., the composition

$$
\left.\left.\left.H^{0}(X, L) \rightarrow L\right|_{p} \oplus L\right|_{q} \rightarrow L^{\prime}\right|_{r}
$$

is zero. Since $h^{0}(L(-p-q))=2$, the above sequence must be exact, so the line corresponding to $L^{\prime}$ is unique (and it is neither of the summands, because $X$ has no $g_{5}^{2}$ ).

It remains to show that exactly five $L \in W_{6}^{2}(X)$ satisfy $h^{0}(L(-p-q))=2$. On a general smooth curve $X$ of genus five, every $g_{6}^{2}$ has the form $\left|\omega_{X}(-D)\right|$ for a unique $D \in X^{(2)}$. The pair $\left(X, \omega_{X}(-p-q)\right)$ is general, so the image of $X$ in $\left|\omega_{X}(-p-q)\right|^{\vee}$ is a nodal sextic [ACG11, XXI, $\left.\S 10\right]$. By the genus formula it has five nodes, so there are five $D \in X^{(2)}$ such that $h^{0}\left(\omega_{X}(-D-p-q)\right)=2$, giving five choices for $L:=\omega_{X}(-D)$.

5.2. First base change. If $T \subset|H|$ is sufficiently general, then Lemma 5.1 implies that there is a Zariski open subset $T^{\circ} \subset T$ such that $\mathcal{C}_{t} \in \mathcal{U}_{6}^{2}$ for all $t \in T^{\circ}$ and $C:=\mathcal{C}_{0}$ is singular for a unique point $0 \in T^{\circ}$. Let $X \stackrel{\nu}{\rightarrow} C$ be the normalization. By an argument analogous to [IW18, Lemma 2.3], we may assume that the five pairs $\left(X, \nu^{*} g_{6}^{2}\right)$ are also general. Let $U:=\mathcal{G}_{6}^{2}\left(\mathcal{C}_{T^{\circ}}\right)$ parameterize nets of degree 6 on the fibers of $\mathcal{C}_{T^{\circ}}:=\mathcal{C} \times_{T} T^{\circ} \rightarrow T^{\circ}$. It is étale of degree 5 over $T^{\circ}$.

5.3. Surfaces of divisors. Each fiber of the family $\mathcal{C}_{U} \rightarrow U$ has a canonical $g_{6}^{2}$. These linear systems form a $\mathbb{P}^{2}$-bundle $\mathcal{P} \rightarrow U$ embedded in the relative symmetric power $\mathcal{C}_{U}^{(6)}$. Put $\mathcal{S}:=$ $\mathcal{P} \times{ }_{\mathcal{C}_{U}^{(6)}} \widetilde{\mathcal{C}}_{U}^{(6)}$

5.4. Second base change. The map $\mathcal{S} \rightarrow U$ naturally factors as $\mathcal{S} \rightarrow \widetilde{U} \rightarrow U$, where $\widetilde{U}$ is the double cover of $U$ parameterizing the connected components of the fibers of $\mathcal{S} \rightarrow U$. From now on we think of $\mathcal{S}$ as a family over $\widetilde{U}$; for instance $\mathcal{S}_{u}:=\mathcal{S} \times_{\widetilde{U}}\{u\}$ is connected for $u \in \widetilde{U}$. The following fact ensures that $\widetilde{U} \rightarrow U$ is unramified, so that $\Theta_{\widetilde{U}}$ is nonsingular:

Lemma 5.2. For every $g_{6}^{2}$ on $C$, the corresponding fiber of $\mathcal{S}$ in $\widetilde{C}^{(6)}$ has two connected components. Proof. One checks (e.g., using (5.1)) that the surface in $\widetilde{C}^{(6)}$ is the image of the surface in $\widetilde{X}^{(6)}$ determined by $\nu^{*} g_{6}^{2}$, which has two connected components. These components are smooth, and their images in $\widetilde{C}^{(6)}$ are disjoint, provided that the image of $C \rightarrow g_{6}^{2 \vee}$ is admissible in the sense of Welters [Wel81, (8.14), (9.2), (9.6)]. This means that the image of $C$ is nodal and that for any line $l$ in $g_{6}^{2 \vee}$, the divisor cut on $l$ by (the image of) $C$ is either reduced or contains exactly one divisor 
of the form $2 P, 3 P$, or $2 P+2 Q$. It is easy to see that a general plane sextic of geometric genus five is admissible $[\mathrm{DH} 88,1(\mathrm{c})]$. Since the five pairs $\left(X, \nu^{*} g_{6}^{2}\right)$ are general, the result follows.

Lemma 5.3. Given $u \in \widetilde{U}$ lying over $0 \in T$, there is a connected Zariski open neighborhood $\widetilde{U}^{\circ} \subseteq \widetilde{U}$ of $u$ such that $\mathcal{S}_{v}$ is irreducible for all $v \in \widetilde{U}^{\circ}$, and smooth unless $v$ lies over 0 . Consequently $\mathcal{S}_{\widetilde{U}^{\circ}}$ is integral.

Proof. By [Wel81, (8.13)], if the image of $X \rightarrow g_{6}^{2} \vee$ is admissible (see the proof of Lemma 5.2), then the special surfaces obtained from $g_{6}^{2}$ in $\tilde{X}^{(6)}$ are smooth. As in the proof of Lemma 5.2, general nodal plane sextics are admissible. The same argument applies to a general curve of genus 6 together with a $g_{6}^{2}$, so $\mathcal{S}_{v}$ is smooth for most $v \in \widetilde{U}$ provided that $T$ and $R$ are sufficiently general. We let $\widetilde{U}^{\circ}$ be the connected component of $\widetilde{U}$ containing $u$ minus the points $v$ where $\mathcal{S}_{v}$ is singular.

Since $\mathcal{S} \rightarrow \mathcal{P}$ is finite, flat and generically étale, $\mathcal{S}$ satisfies Serre's conditions $R_{0}$ and $S_{1}$ (in fact it is Cohen-Macaulay), so $\mathcal{S}$ is reduced. The previous paragraph then implies that $\mathcal{S}_{\widetilde{U}^{\circ}}$ is integral.

5.5. Third base change. In order to embed the surfaces we constructed into the theta divisors of our family, we need to choose lifts of divisors of the residual $g_{4}^{1}$ that we will then add to lifts of the divisors of the $g_{6}^{2}$ to obtain lifts of canonical divisors. For this we need to introduce a third base change, defined as follows. Taking the $g_{4}^{1}$ residual to each $g_{6}^{2}$ determines, in a completely analogous way, a family $\mathcal{D} \rightarrow \widetilde{U}$ of 1 -dimensional special subvarieties in $\widetilde{\mathcal{C}}_{\widetilde{U}}^{(4)}$. The morphism $C \rightarrow g_{4}^{1 \vee}$ determined by the image $g_{6}^{2}=K_{C}-g_{4}^{1} \in U$ of $u \in \widetilde{U}$ is generically unramified, so $\mathcal{D}_{u} \rightarrow g_{4}^{1}$ is also generically unramified, which means $\mathcal{D}_{u}$ is generically smooth. Given a general divisor $D \in \mathcal{D}_{u}$, there is a connected curve $\widetilde{T} \subset \mathcal{D}_{\widetilde{U}^{\circ}}$ containing $D$ such that $\widetilde{T} \rightarrow \widetilde{U}^{\circ}$ is étale. Such a curve can be obtained, for instance, by choosing a hyperplane section of $\mathcal{D}$ (in some projective embedding) which meets the smooth locus of $\mathcal{D}_{u}$ transversely, then removing the ramification locus from a component which contains $D$.

5.6. The family of surfaces. The natural embedding of $\mathcal{S}_{\widetilde{T}}$ in $\widetilde{\mathcal{C}}_{\widetilde{T}}^{(10)}$ induces a rational map $\mathcal{S}_{\widetilde{T}} \rightarrow$ $\Theta_{\widetilde{T}}$, defined on the open subset $\mathcal{S}^{\circ} \subset \mathcal{S}_{\widetilde{T}}$ of divisors avoiding the nodes. Note that $\mathcal{S}_{E}^{\circ} \neq \varnothing$ for all $E \in \widetilde{T}$, and $\mathcal{S}_{E}^{\circ}=\mathcal{S}_{E}$ unless $E$ lies over $0 \in T$. There is a dense open subset $\widetilde{T}^{\circ} \subset \widetilde{T}$ such that the image of the birational morphism $\mathcal{S}_{t} \rightarrow \Theta_{t}$ is smooth for all $t \in \widetilde{T}^{\circ}$. Since $\mathcal{S} \rightarrow U$ is flat, the image of $\mathcal{S}_{\widetilde{T}^{\circ}} \rightarrow \Theta_{\widetilde{T}^{\circ}}$ is flat over $\widetilde{T}^{\circ}$. The (scheme-theoretic) closure $\mathcal{V}$ of the image of $\mathcal{S}^{\circ}$ in $\Theta_{\widetilde{T}}$ agrees with that of $\mathcal{S}_{\widetilde{T}^{\circ}}$, because $\mathcal{S}^{\circ}$ is integral. In particular $\mathcal{V} \rightarrow \widetilde{T}$ is flat [Har77, III, 9.8].

\section{A NODAL FIBER}

In this section we determine the fiber $V \subset \Theta_{0}$ of $\mathcal{V}$ (see Paragraph 5.6) over $D \in \widetilde{T}$. 
6.1. Notation. Let $P \rightarrow A_{0}$ and $\widetilde{B} \rightarrow \Theta_{0}$ be the normalizations. Recall from Paragraph 4.3 that $P \rightarrow B$ is a $\mathbb{P}^{1}$-bundle with distinguished sections $B_{0}$ and $B_{\infty}$, and $\widetilde{B} \rightarrow B$ is the blowup along $\Xi \cap \Xi_{\beta}$ for the extension datum $\beta \in B$. If $p, q \in \widetilde{X}$ lie over one of the nodes of $\widetilde{C}$, we may assume that $\beta=\left[p, q^{\prime}\right]$ (the other choice $\left[p^{\prime}, q\right]$ corresponds to relabelling $B_{0}$ and $B_{\infty}$ ) [IW18, Proposition 1.5].

There is a unique divisor in the pencil $\left|\pi_{*} D\right|$ passing through the node of $C$; the corresponding divisor on the normalization $X$ can be written as $\pi_{*} E$ where $E=p+q^{\prime}+u+v$ for some $u, v \in \widetilde{X}$. Since $D$ is general it can be identified with a divisor on $\widetilde{X}$. Replacing $v$ by $v^{\prime}$ if necessary, we may assume that $D$ and $E$ belong to the same curve $\widetilde{Y} \subset \widetilde{X}^{(4)}$ (among the two curves tetragonally related to $\tilde{X}$ via $\left.\left|\pi_{*} D\right|\right)$. The divisor $F:=p+q^{\prime}+u^{\prime}+v^{\prime}$ also belongs to $\tilde{Y}$. Let $d, e, f \in \tilde{Y}$ correspond to $D, E, F \in \widetilde{X}^{(4)}$. If $g_{Y}$ denotes the $g_{4}^{1}$ on $Y$, then $h^{0}\left(g_{Y}-\pi_{*}(e+f)\right)>0$.

Lemma 6.1. The special subvariety $V_{d+e+f}$ is smooth.

Proof. Set $g_{5}^{1}:=\left|\omega_{Y}\left(-\pi_{*}(d+e+f)\right)\right|$, and suppose for a moment that $Y \rightarrow g_{5}^{1 \vee}$ is a well-defined morphism with only simple ramification. By Welters' criterion [Wel81, (8.13)], the associated special subvarieties $S_{1}, S_{2} \subset \tilde{Y}^{(5)}$ are smooth. We may assume that $V_{d+e+f}$ is the image of $S_{1}$. If some pencil in $\tilde{Y}^{(5)}$ meets $S_{1}$, its image in $Y^{(5)}$ must be $g_{5}^{1}$, so the pencil must be all of $S_{1}$. This is not possible: if a divisor $p_{1}+\ldots+p_{5}$ belongs to $S_{1}$, so does $p_{1}^{\prime}+p_{2}^{\prime}+\ldots+p_{5}$, hence $p_{1}+p_{2}$ is linearly equivalent to $p_{1}^{\prime}+p_{2}^{\prime}$ and $\widetilde{Y}$ is hyperelliptic, which contradicts our genericity assumptions. Therefore $S_{1}$ maps isomorphically onto $V_{d+e+f}$.

It remains to show that $Y \rightarrow g_{5}^{1 \vee}$ has simple ramification. For this, we just need the pair $\left(Y, g_{5}^{1}\right)$, or equivalently $\left(Y, \pi_{*}(d+e+f)\right)$, to be sufficiently general [ACG11, XXI, (11.9)].

The data $\left(X, \pi_{*}(p+q)\right)$ is in finite correspondence with the data $\left(Y, \pi_{*}(e+f)\right)$. So, for a general choice of $\left(X, \pi_{*}(p+q)\right),\left(Y, \pi_{*}(e+f)\right)$ will also be general. We can then choose $d$ to be a general point on $\tilde{Y}$.

6.2. The central fiber. We will show that $V$ is birational to

$$
W:=\Xi_{[d, e]} \cap \Xi_{[d, f]}=\left(\Xi \cap \Xi_{[u, v]}\right)+[d, f] \subset B
$$

(see Paragraph 1.6). Since $X$ is general, we may assume that $[u, v],\left[p, q^{\prime}\right] \in B$ are too [Iza95, 4.6]. This implies that $W$ and $\Xi \cap \Xi_{\beta}$ are smooth by a Bertini-style argument.

Lemma 6.2. The projection $\widetilde{B} \rightarrow B$ induces isomorphisms

$$
\begin{gathered}
\widetilde{W} \rightarrow W, \\
\Delta \cap \widetilde{W} \rightarrow V_{d+e+f}, \\
\widetilde{\Xi} \cap \widetilde{W} \rightarrow W_{d}, \\
\widetilde{\Xi}_{\beta} \cap \widetilde{W} \rightarrow W_{d}+\beta,
\end{gathered}
$$


where $\Delta \subset \widetilde{B}$ is the exceptional divisor and $\widetilde{Z} \subset \widetilde{B}$ denotes the proper transform whenever $Z \subset B$. Proof. Note that $W_{d}$ and $W_{d}+\beta$ are the embeddings of $\widetilde{Y}_{\lambda}$ in $W$, as $\beta=[e, f]$ (see Paragraph 1.6). By Proposition 3.4, $W_{d} \cap\left(W_{d}+\beta\right)=\varnothing$. It follows by Lemma 1.2(b) and (c) that

$$
\Xi \cap \Xi_{\beta} \cap W=\Xi \cap \Xi_{[e, f]} \cap \Xi_{[d, e]} \cap \Xi_{[d, f]}=\left(V_{d+e+f} \cup W_{d}\right) \cap\left(V_{d+e+f} \cup W_{d}+\beta\right)=V_{d+e+f} .
$$

Next note that

$$
W=\left(W \backslash W_{d}\right) \cup\left(W \backslash W_{d}+\beta\right)
$$

and

$$
V_{d+e+f}=\left(V_{d+e+f} \backslash W_{d}\right) \cup\left(V_{d+e+f} \backslash W_{d}+\beta\right) .
$$

Now $V_{d+e+f} \backslash W_{d}$ is a Cartier divisor in $W \backslash W_{d}$, and $V_{d+e+f} \backslash W_{d}+\beta$ is a Cartier divisor in $W \backslash W_{d}+\beta$. This implies that $\Xi \cap \Xi_{\beta} \cap W=V_{d+e+f}$ is a Cartier divisor in $W$, giving the first two isomorphisms. Moreover $(\widetilde{\Xi} \cap \widetilde{W}) \backslash \Delta \rightarrow(\Xi \cap W) \backslash\left(\Xi \cap \Xi_{\beta}\right)=W_{d} \backslash V_{d+e+f}$ is an isomorphism. Since $V_{d+e+f}$ is smooth, $\Xi$ intersects $W$ transversely along $V_{d+e+f} \backslash W_{d}$, which means $\widetilde{\Xi} \widetilde{W}=\widetilde{W} \cong \stackrel{\cong}{\rightarrow} W_{d}$ as sets and also generically as schemes. Next, the isomorphism holds scheme-theoretically everywhere because $\widetilde{\Xi} \cap \widetilde{W}$ is a generically smooth Cartier divisor in the smooth variety $\widetilde{W}$, so it is reduced. The fourth isomorphism is similar.

Lemma 6.3. $V$ contains the image $\bar{W} \subset \Theta_{0}$ of $\widetilde{W} \subset \widetilde{B}$.

Proof. First note that $W=\left(\Xi \cap \Xi_{[u, v]}\right)+[d, f]=V_{u+v}+\mathcal{O}_{\widetilde{X}}\left(D-F^{\prime}\right)$ by Lemma $1.2($ a) and Paragraph 1.6. Thus, a general point $\bar{L} \in \bar{W}$ corresponds to exactly one $L \in W \backslash V_{d+e+f}$, which can be represented by

$$
G+u+v+D-F^{\prime}=G+D-p^{\prime}-q
$$

for some divisor $G \in \widetilde{X}^{(6)}$ supported away from the nodes with $\pi_{*} G \in \nu^{*} g_{6}^{2}$. By abuse of notation we can think of $G$ as a divisor on $\widetilde{C}$, in which case $\pi_{*} G \in g_{6}^{2}$ and $G+D \in \mathcal{S}^{\circ}$.

Let $\Theta_{0}^{\circ} \cong \widetilde{B} \backslash\left(\widetilde{\Xi} \cup \widetilde{\Xi}_{\beta}\right)$ be the smooth locus of $\Theta_{0}$. The map $\Theta_{0}^{\circ} \hookrightarrow \widetilde{B} \rightarrow B$ is induced by $\nu^{*}: \operatorname{Pic}^{0}(\widetilde{C}) \rightarrow \operatorname{Pic}^{0}(\widetilde{X})$. By choosing appropriate theta characteristics on $\widetilde{C}$ and $\widetilde{X}$, it can be taken to send $\mathcal{O}_{\widetilde{C}}(G+D) \in V$ to $L \in W$ (we could have chosen $G+D-p-q^{\prime}$ instead; however $G+D-p-q$ and $G+D-p^{\prime}-q^{\prime}$ have the wrong parity). It follows that $\mathcal{O}_{\widetilde{C}}(G+D)=\bar{L}$. This shows that $V$ contains an open subset of $\bar{W}$, and hence all of $\bar{W}$.

Lemma 6.4. The Hilbert polynomial of $V$ is $\chi\left(\mathcal{O}_{V}\left(n \Theta_{0}\right)\right)=20 n^{2}-40 n+22$.

Proof. Since $\mathcal{V} \rightarrow \widetilde{T}$ is flat, it suffices to compute the Hilbert polynomial of $\mathcal{V}_{t}$ for any $t \in \widetilde{T}^{\circ}$. For simplicity set $Z:=\mathcal{C}_{t}$ and let $G \in \widetilde{Z}^{(4)}$ correspond to $t$, so that $\mathcal{V}_{t}=V_{G}$. The special surface $S \subset \widetilde{Z}^{(6)}$ associated to $g_{4}^{1}:=\left|\pi_{*} G\right|$ has two components; let $S_{1}$ be the one whose image is $V_{G}$. Since $S_{1} \rightarrow V_{G}$ is a birational morphism between smooth varieties, their Hilbert polynomials are 
the same. We will show that $\chi\left(\mathcal{O}_{S}\left(n \Theta_{t}\right)\right)=40 n^{2}-80 n+44$, which gives the result (after some algebra) because the calculation also works on each of the two curves tetragonally related to $Z$ via $g_{4}^{1}$. Since $\chi\left(\mathcal{O}_{S}\left(2 n \Theta_{t}\right)\right)=\chi\left(\mathcal{O}_{S}(n \widetilde{\Theta})\right)$ for all $n \in \mathbb{N}$, where $\widetilde{\Theta}$ is a theta divisor for $\operatorname{Pic}^{6}(\widetilde{Z})$, it suffices to show that $\chi\left(\mathcal{O}_{S}(n \widetilde{\Theta})\right)=160 n^{2}-160 n+44$.

Let $\iota: g_{6}^{2} \hookrightarrow Z^{(6)}$ be the embedding of the $g_{6}^{2}$ residual to $g_{4}^{1}$, with $\rho: S \rightarrow g_{6}^{2}$ and $\widetilde{\iota}: S \hookrightarrow \widetilde{Z}^{(6)}$ the associated projections. Since $\pi^{(6)}: \widetilde{Z}^{(6)} \rightarrow Z^{(6)}$ is an affine morphism, $\pi_{*}^{(6)}$ commutes with arbitrary base change. In particular $\iota^{*} \pi_{*}^{(6)} H=\rho_{*} \widetilde{\iota}^{*} H$ for $H:=\mathcal{O}_{\widetilde{Z}(6)}(n \widetilde{\Theta})$. Since $R^{i} \rho_{*}=0$ for $i>0$

$$
\chi\left(\mathcal{O}_{S}(n \widetilde{\Theta})\right)=\chi\left(\iota^{*} \pi_{*}^{(6)} H\right)=\int_{|L|} \operatorname{ch}\left(\iota^{*} \pi_{*}^{(6)} H\right) \operatorname{td}(|L|)=\int_{|L|} \iota^{*} \operatorname{ch}\left(\pi_{*}^{(6)} H\right) \operatorname{td}(|L|) .
$$

By Grothendieck-Riemann-Roch

$$
\operatorname{ch}\left(\pi_{*}^{(6)} H\right)=\pi_{*}^{(6)}\left(\operatorname{ch}(H) \operatorname{td}\left(\widetilde{Z}^{(6)}\right)\right) \cdot \operatorname{td}\left(Z^{(6)}\right)^{-1} .
$$

The Chern classes of symmetric products are well-known [ACGH85, VII, (5.4)]. In particular

$$
c\left(\widetilde{Z}^{(6)}\right)=1-4 \widetilde{\eta}-\widetilde{\theta}+10 \widetilde{\eta}^{2}+5 \widetilde{\eta} \widetilde{\theta}+\frac{1}{2} \widetilde{\theta}^{2}+\cdots,
$$

(see Lemma 8.1 for notation) and hence

$$
\operatorname{td}\left(\widetilde{Z}^{(6)}\right)=1-2 \widetilde{\eta}-\frac{1}{2} \widetilde{\theta}+\frac{13}{6} \widetilde{\eta}^{2}+\frac{13}{12} \widetilde{\eta} \widetilde{\theta}+\frac{1}{8} \widetilde{\theta}^{2}+\cdots
$$

Since

$$
\operatorname{ch}(H)=1+n \widetilde{\theta}+\frac{1}{2} n^{2} \widetilde{\theta}^{2}+\cdots,
$$

it follows that

$$
\operatorname{ch}(H) \operatorname{td}\left(\widetilde{Z}^{(6)}\right)=1-2 \widetilde{\eta}+\left(n-\frac{1}{2}\right) \widetilde{\theta}+\frac{13}{6} \widetilde{\eta}^{2}+\left(-2 n+\frac{13}{12}\right) \widetilde{\eta} \widetilde{\theta}+\left(\frac{1}{2} n^{2}-\frac{1}{2} n+\frac{1}{8}\right) \widetilde{\theta}^{2}+\cdots,
$$

so by Lemma 8.1

$$
\begin{aligned}
\pi_{*}^{(6)}\left(\operatorname{ch}(H) \operatorname{td}\left(\widetilde{Z}^{(6)}\right)\right) & =64+(160 n-144) \eta+(32 n-16) \theta+\left(160 n^{2}-320 n+\frac{484}{3}\right) \eta^{2} \\
& +\left(80 n^{2}-112 n+\frac{112}{3}\right) \eta \theta+\left(8 n^{2}-8 n+2\right) \theta^{2}+\cdots
\end{aligned}
$$

Again a general formula gives

$$
c\left(Z^{(6)}\right)=1+\eta-\theta+\frac{1}{2} \theta^{2}+\cdots
$$

and hence

$$
\operatorname{td}\left(Z^{(6)}\right)^{-1}=1-\frac{1}{2} \eta+\frac{1}{2} \theta+\frac{1}{6} \eta^{2}-\frac{1}{3} \eta \theta+\frac{1}{8} \theta^{2}+\cdots
$$


Using (6.2)

$$
\begin{aligned}
\operatorname{ch}\left(\pi_{*}^{(6)} H\right) & =64+(160 n-176) \eta+(32 n+16) \theta+\left(160 n^{2}-400 n+244\right) \eta^{2} \\
& +\left(80 n^{2}-48 n-48\right) \eta \theta+\left(8 n^{2}+8 n+2\right) \theta^{2}+\cdots
\end{aligned}
$$

The class of a linear system in $Z^{(d)}$ can be computed using a special case of the secant plane formula [ACGH85, VIII, (3.2)]. After simplifying (using [Mac62, (6.3)]), the class of $|L|$ in $Z^{(6)}$ is

$$
10 \eta^{4}-4 \eta^{3} \theta+\frac{1}{2} \eta^{2} \theta^{2}
$$

It follows that the degree of $\iota^{*} \operatorname{ch}\left(\pi_{*}^{(6)} H\right)$ is $160 n^{2}-400 n+244$. The class of a pencil in $|L|$ is

$$
-5 \eta^{5}+\eta^{4} \theta
$$

so the intersection of $\iota^{*} \operatorname{ch}\left(\pi_{*}^{(6)} H\right)$ with a line has degree $160 n-176$. The class of a point is obviously $\eta^{6}$, so the codimension 0 term of $\iota^{*} \operatorname{ch}\left(\pi_{*}^{(6)} H\right)$ has degree 64 . Therefore

$$
\iota^{*} \operatorname{ch}\left(\pi_{*}^{(6)} H\right)=64+(160 n-176) h+\left(160 n^{2}-400 n+244\right) h^{2},
$$

where $h:=c_{1}\left(\mathcal{O}_{|L|}(1)\right)$. Finally $(6.1)$ gives

$$
\begin{aligned}
\chi\left(\mathcal{O}_{S}(n \widetilde{\Theta})\right) & =\int_{|L|}\left(64+(160 n-176) h+\left(160 n^{2}-400 n+244\right) h^{2}\right)\left(1+\frac{3}{2} h+h^{2}\right) \\
& =\int_{|L|} 64+(160 n-80) h+\left(160 n^{2}-160 n+44\right) h^{2} \\
& =160 n^{2}-160 n+44,
\end{aligned}
$$

as required.

Proposition 6.5. $V=\bar{W}$.

Proof. Since $\bar{W} \subseteq V$, it suffices to show that the Hilbert polynomial of $\bar{W}$ is also $20 n^{2}-40 n+22$. The projection $\widetilde{B} \rightarrow B$ factors through $P \stackrel{\varphi}{\rightarrow} B$. Moreover $\widetilde{B}$ moves in a pencil of divisors on $P$ spanned by $B_{0}+\varphi^{-1}\left(\Xi_{\beta}\right)$ and $B_{\infty}+\varphi^{-1}(\Xi)[\mathrm{IW} 18,1.2]$. Therefore $\widetilde{B}$ is polarized by

$$
\left.\left(B_{0}+\varphi^{-1}\left(\Xi_{\beta}\right)\right)\right|_{\widetilde{B}}=\widetilde{\Xi}+\widetilde{\Xi}_{\beta}+\Delta .
$$

This restricts to a divisor on $\widetilde{W}$, which, by Lemma 6.2 , can be identified with the divisor

$$
\Phi:=W_{d}+\left(W_{d}+\beta\right)+V_{d+e+f}=W_{d}+\left(\Xi_{\beta} \cap W\right)
$$

on $W$. Write $\xi$ for the restriction of $[\Xi]$ to $W$, considered as an algebraic equivalence class, and let $\zeta$ be the class of a point. Note that $\xi^{2}=4 ! \zeta=24 \zeta$. The normal bundle sequence for $W \hookrightarrow B$ gives

$$
\operatorname{td}(W)=\operatorname{td}\left(\mathcal{O}_{B}(\Xi)\right)^{-2}=\left(1+\frac{\xi}{2}+2 \zeta\right)^{-2}=1-\xi+14 \zeta .
$$


By Lemma 1.2(d) and (6.3), $[\Phi]=\xi+\omega$, where $\omega:=\left[W_{d}\right]$. Therefore

$$
\frac{1}{2}[\Phi]^{2}=\frac{1}{2}(24 \zeta+2 \xi \omega)=12 \zeta+\xi \omega=20 \zeta
$$

(see Theorem 2.1), which gives

$$
\operatorname{ch}\left(\mathcal{O}_{W}(n \Phi)\right) \operatorname{td}(W)=\left(1+(\xi+\omega) n+20 \zeta n^{2}\right)(1-\xi+14 \zeta) .
$$

The coefficient of $\zeta$ in this expression is

$$
\chi\left(\mathcal{O}_{W}(n \Phi)\right)=20 n^{2}-(\xi+\omega) \xi n+14=20 n^{2}-32 n+14 .
$$

The quotient map $W \stackrel{\psi}{\rightarrow} \bar{W}$ identifies the disjoint curves $W_{d}$ and $W_{d}+\beta$. There is a short exact sequence

$$
0 \rightarrow \mathcal{O}_{\bar{W}} \rightarrow \psi_{*} \mathcal{O}_{W} \rightarrow \psi_{*} \mathcal{O}_{W_{d}} \rightarrow 0
$$

Twisting by $\Psi:=\left.\Theta_{0}\right|_{\bar{W}}$ and using the projection formula gives

$$
0 \rightarrow \mathcal{O}_{\bar{W}}(n \Psi) \rightarrow \psi_{*} \mathcal{O}_{W}(n \Phi) \rightarrow \psi_{*} \mathcal{O}_{W_{d}}(n \Phi) \rightarrow 0 .
$$

Since $n \Phi$ has degree $8 n$ on the genus 9 curve $W_{d}$, the Hilbert polynomial of $\bar{W}$ is

$$
20 n^{2}-32 n+14-(8 n-8)=20 n^{2}-40 n+22,
$$

as required.

Lemma 6.6. If $\xi:=[\Xi]$ and $\omega:=\left[W_{d}\right]$, then

$$
[\widetilde{W}]=\left(\xi^{2}, \xi-\omega\right) \text { in } \mathrm{CH}_{2}^{\mathrm{alg}}(\widetilde{B})=\mathrm{CH}_{2}^{\mathrm{alg}}(B) \oplus \mathrm{CH}_{1}^{\mathrm{alg}}\left(\Xi \cap \Xi_{\beta}\right),
$$

where $\mathrm{CH}_{d}^{\text {alg }}$ is the group of $d$-dimensional algebraic cycles modulo algebraic equivalence. Consequently $[\widetilde{W}]^{2}=16$.

Proof. Let $\varphi: \widetilde{B} \rightarrow B$ be the blowup and $\psi: \Delta \rightarrow \Xi \cap \Xi_{\beta}$ its restriction to $\Delta$. By standard properties of blowing up [Bea77b, 0.1.3.ii]

$$
[\widetilde{W}]=\left(\varphi_{*}[\widetilde{W}], \psi_{*}\left(\left.[\widetilde{W}]\right|_{\Delta}\right)\right)=\left([W],\left[V_{d+e+f}\right]\right)=\left(\xi^{2}, \xi-\omega\right) .
$$

Therefore, by Proposition 3.4,

$$
[\widetilde{W}]^{2}=\int_{B} \xi^{4}+\int_{\Delta} c_{1}\left(\mathcal{O}_{\Delta}(\Delta)\right) \psi^{*}(\xi-\omega)^{2}=4 !-\int_{\Xi \cap \Xi_{\beta}} \xi^{2}-2 \xi \omega+\omega^{2}=2 \int_{B} \frac{\xi^{4}}{3}=16,
$$

as required. 


\section{Proof of THE MAIN THEOREM}

Proposition 7.1. If $t \in \widetilde{T}$ and $\Theta_{t}$ is smooth, then $\left[\mathcal{V}_{t}\right]^{2}=16$.

Proof. The class $[\mathcal{V}] \in \mathrm{CH}_{3}\left(\Theta_{\widetilde{T}}\right)$ defines a family of 0-cycle classes (i.e., a 1-cycle) $[\mathcal{V}]^{2} \in \mathrm{CH}_{1}\left(\Theta_{\widetilde{T}}\right)$ over $\widetilde{T}$, and $\left[\mathcal{V}_{t}\right]^{2}$ is the degree of the specialization of $[\mathcal{V}]^{2}$ at $t\left[\right.$ Ful 84, 10.1]. Since $\Theta_{\widetilde{T}} \rightarrow \widetilde{T}$ is flat, specialization at $t$ is the same as restricting to $\Theta_{t}$, for any $t \in \widetilde{T}$. We can specialize $[\mathcal{V}]^{2}$ to the central fiber, but since $\Theta_{0}$ is singular the meaning of $[V]^{2}$ is not clear.

To remedy this, we pass to the operational Chow rings $\mathrm{CH}^{*}\left(\Theta_{D}\right)$ and $\mathrm{CH}^{*}\left(\Theta_{\widetilde{T}}\right)$, which act on $\mathrm{CH}_{*}\left(\Theta_{D}\right)$ and $\mathrm{CH}_{*}\left(\Theta_{\widetilde{T}}\right)$ via cap product. There is a unique "Poincaré dual" $\nu \in \mathrm{CH}^{2}\left(\Theta_{\widetilde{T}}\right)$ such that $\nu \cap\left[\Theta_{\tilde{T}}\right]=[\mathcal{V}][$ Ful84, 17.4]. Using the cap product on Chow groups [Ful84, 8.1] for the inclusion $\iota: \Theta_{0}=\Theta_{D} \hookrightarrow \Theta_{\widetilde{T}}$, one checks that $\iota^{*} \nu \cap\left[\Theta_{D}\right]=[V]$ and $\iota^{*} \nu^{2} \cap\left[\Theta_{D}\right]=\iota^{*}[\mathcal{V}]^{2}$. It follows that

$$
\int_{\Theta_{t}}\left[\mathcal{V}_{t}\right]^{2}=\int_{\Theta_{D}} \iota^{*}[\mathcal{V}]^{2}=\int_{\Theta_{D}} \iota^{*} \nu \cap[V]=\int_{\Theta_{D}} \iota^{*} \nu \cap \psi_{*}[\widetilde{W}]=\int_{\widetilde{B}} \psi^{*} \iota^{*} \nu \cap[\widetilde{W}]=\int_{\widetilde{B}}(\iota \psi)^{*}[\mathcal{V}] \cdot[\widetilde{W}],
$$

where $\psi: \widetilde{B} \rightarrow \Theta_{0}$ denotes the normalization. If $\delta:=(\iota \psi)^{*}[\mathcal{V}]-[\widetilde{W}]$ then

$$
\psi_{*} \delta=\psi_{*}\left(\psi^{*} \iota^{*} \nu \cap[\widetilde{B}]\right)-\psi_{*}[\widetilde{W}]=\iota^{*} \nu \cap\left[\Theta_{D}\right]-[V]=0 .
$$

Let $U:=\widetilde{B} \backslash\left(\widetilde{\Xi} \amalg \widetilde{\Xi}_{\beta}\right)$ be the smooth locus of $\Theta_{0}$, and consider the localization sequences

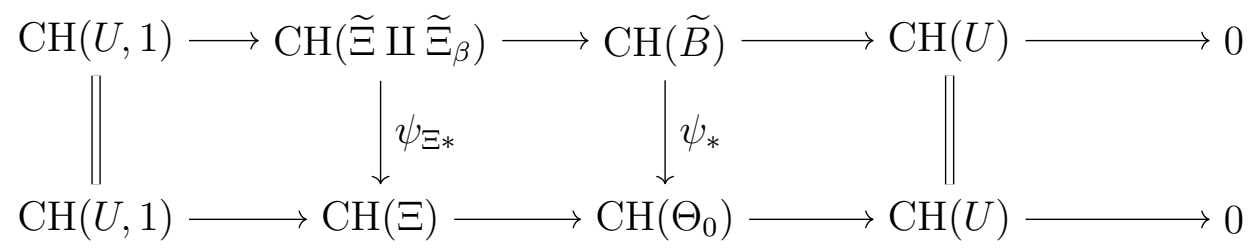

(here $\mathrm{CH}(U, 1)$ is one of Bloch's higher Chow groups [Blo94], but all we need is a group depending only on $U$, which is easy to construct with a little thought). Since $\psi_{*} \delta=0$, the diagram implies $\left.\delta\right|_{U}=0$, hence one can find $\gamma \in \mathrm{CH}_{2}\left(\widetilde{\Xi} \amalg \widetilde{\Xi}_{\beta}\right)$ mapping to $\delta \in \mathrm{CH}_{2}(\widetilde{B})$. After possibly subtracting an element of $\mathrm{CH}(U, 1)$, we may assume that $\psi_{\Xi *} \gamma=0 \in \mathrm{CH}_{2}(\Xi)$. Lemma 6.2 implies that

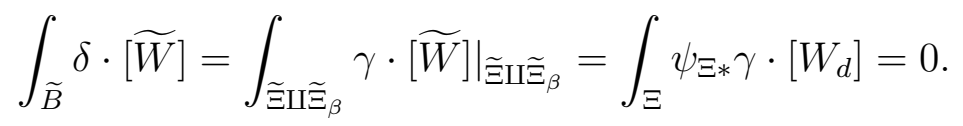

Therefore $\left[\mathcal{V}_{t}\right]^{2}=[\widetilde{W}]^{2}=16$, as required.

Proof of Theorem 1. For a very general ppav $(A, \Theta)$, one constructs a degeneration as above to get $\left[\widetilde{X}_{\lambda}\right]^{2}=16$ for every Prym-curve $X$. The other intersection numbers are given by Theorem 2.1.

If $X$ and $Y$ are Prym-curves corresponding to lines $E$ and $F$ on a cubic surface, then Theorem 2.1 says that $\left[\widetilde{X}_{\lambda}\right] \cdot\left[\widetilde{Y}_{\lambda}\right]=2(7-E . F)$. Choose mutually unrelated Prym-curves $X_{1}, \ldots, X_{6}$ and another one $Y$ related to two of the $X_{i}$. The argument of Corollary 3.5 shows that the lattice $\mathbb{L}$ spanned by classes of the form $\left[\widetilde{X}_{i \lambda}\right]-\left[\widetilde{Y}_{\lambda}\right]$ is isometric to $\mathrm{E}_{6}(2)$, and therefore spans $\mathbb{K}_{\mathbb{Q}}^{+}$. It is straightforward to 
check, e.g., by the nondegeneracy of the pairing, that $\mathbb{L}$ contains $\left[\widetilde{X}_{\lambda}\right]-\left[\widetilde{Z}_{\lambda}\right]$ for any two Prym-curves $X$ and $Z$. The $\mathbb{Q}$-vector space spanned by the $\left[\widetilde{X}_{\lambda}\right]$ is at least 7 -dimensional, as $\left[\widetilde{X}_{\lambda}\right] \notin \mathbb{K}$, so it has to be $\mathbb{Q}[\Theta]^{2}+\mathbb{K}_{\mathbb{Q}}^{+}$.

\section{ApPEndix}

8.1. Cohomological Calculations. Let $X$ be a smooth curve of genus 6 and $\widetilde{X} \stackrel{\pi}{\rightarrow} X$ an étale double cover.

Lemma 8.1. Let $\eta, \theta \in H^{2}\left(X^{(6)}, \mathbb{Z}\right)$ be, respectively, the class of $X^{(5)}$ (plus a point), and the class of the polarization inherited from $\operatorname{Pic}^{6}(X)$. If $\widetilde{\eta}, \widetilde{\theta} \in H^{2}\left(\widetilde{X}^{(6)}, \mathbb{Z}\right)$ are the corresponding classes for $\widetilde{X}$, then $\pi_{*}^{(6)}: H^{*}\left(\widetilde{X}^{(6)}, \mathbb{Z}\right) \rightarrow H^{*}\left(X^{(6)}, \mathbb{Z}\right)$ has the following description:

\begin{tabular}{|c|c|c|c|c|c|}
\hline$\alpha$ & $\widetilde{\eta}$ & $\widetilde{\theta}$ & $\widetilde{\eta}^{2}$ & $\widetilde{\eta} \widetilde{\theta}$ & $\widetilde{\theta}^{2}$ \\
\hline$\pi_{*}^{(6)} \alpha$ & $32 \eta$ & $32(5 \eta+\theta)$ & $16 \eta^{2}$ & $16 \eta(5 \eta+\theta)$ & $16\left(20 \eta^{2}+10 \eta \theta+\theta^{2}\right)$ \\
\hline
\end{tabular}

Proof. Let $\left(\lambda_{1}, \mu_{1}, \ldots, \lambda_{6}, \mu_{6}\right)$ be a symplectic basis for $H^{1}(X, \mathbb{Z})$ (with $\lambda_{i} \cdot \mu_{i}=1$ for all $i$ ). Using the picture in [BL04, Proposition 12.4.2] we can construct a symplectic basis

$$
\widetilde{\lambda}_{1}, \widetilde{\mu}_{1}, \lambda_{2}^{+}, \mu_{2}^{+}, \lambda_{2}^{-}, \mu_{2}^{-}, \ldots, \lambda_{6}^{+}, \mu_{6}^{+}, \lambda_{6}^{-}, \mu_{6}^{-}
$$

for $H^{1}(\tilde{X}, \mathbb{Z})$ such that $\pi_{*}: H^{1}(\widetilde{X}, \mathbb{Z}) \rightarrow H^{1}(X, \mathbb{Z})$ sends $\widetilde{\lambda}_{1} \mapsto 2 \lambda_{1}$ and $\widetilde{\mu}_{1} \mapsto \mu_{1}$, while the $\lambda_{i}^{ \pm}$and $\mu_{i}^{ \pm}$are sent to $\lambda_{i}$ and $\mu_{i}$ respectively. Let $\rho_{k}: X^{6} \rightarrow X$ be the projections, and note that

$$
\sum_{k=1}^{6} \rho_{k}^{*} \lambda_{i}, \sum_{k=1}^{6} \rho_{k}^{*} \mu_{i} \text { and } \sum_{k=1}^{6} \rho_{k}^{*}\left(\lambda_{i} \mu_{i}\right)
$$

all descend to classes in $H^{*}\left(X^{(6)}, \mathbb{Z}\right)$. We denote the first two by $\xi_{i}$ and $\zeta_{i}$ respectively; the latter is independent of $i$ and descends to $\eta$ [Mac62, (3.1) and (14.2)]. Moreover $\theta=\sum_{i=1}^{6} \sigma_{i}$, where $\sigma_{i}:=\xi_{i} \zeta_{i}$. Similarly $\widetilde{\theta}$ is the sum of $\widetilde{\sigma}_{1}:=\widetilde{\xi}_{1} \widetilde{\zeta}_{1}$ and the $\sigma_{i}^{ \pm}:=\xi_{i}^{ \pm} \zeta_{i}^{ \pm}$.

Since $H^{*}\left(X^{(6)}, \mathbb{Z}\right)$ is torsion-free $[\operatorname{Mac} 62,(12.3)]$, while $H^{*}\left(X^{(6)}, \mathbb{Z}\right) \hookrightarrow H^{*}\left(X^{6}, \mathbb{Z}\right) \rightarrow H^{*}\left(X^{(6)}, \mathbb{Z}\right)$ is multiplication by 6 ! (and likewise for $\widetilde{X}$ ), we can compute $\pi_{*}^{(6)}$ using

$$
\pi_{*}^{\times 6}: H^{*}\left(\widetilde{X}^{6}, \mathbb{Z}\right) \rightarrow H^{*}\left(X^{6}, \mathbb{Z}\right)
$$

Note that the cross product is natural for morphisms of even relative (real) dimension (up to a sign in general) [Spa94, 5.3.10 and 5.6.21]. In particular $\pi_{*}^{(6)} \widetilde{\eta}$ corresponds to

$$
\sum_{k=1}^{6} \pi_{*}^{\times 6} \widetilde{\rho}_{k}^{*}\left(\widetilde{\lambda}_{1} \widetilde{\mu}_{1}\right)=\sum_{k=1}^{6} \rho_{1}^{*}\left(\pi_{*} 1\right) \cdots \rho_{k}^{*}\left(\pi_{*}\left(\widetilde{\lambda}_{1} \widetilde{\mu}_{1}\right)\right) \cdots \rho_{6}^{*}\left(\pi_{*} 1\right)=32 \sum_{k=1}^{6} \rho_{k}^{*}\left(\lambda_{1} \mu_{1}\right),
$$


where $\widetilde{\rho}_{k}: \widetilde{X}^{6} \rightarrow \widetilde{X}$ are the projections. Therefore $\pi_{*}^{(6)} \widetilde{\eta}=32 \eta$. Similarly $\pi_{*}^{(6)} \widetilde{\sigma}_{1}=32 \sigma_{1}$, because

$$
\begin{aligned}
\sum_{k=1}^{6} \sum_{l=1}^{6} \pi_{*}^{\times 6}\left(\widetilde{\rho}_{k}^{*} \widetilde{\lambda}_{1} \widetilde{\rho}_{l}^{*} \widetilde{\mu}_{1}\right) & =\sum_{k=1}^{6}\left(\pi_{*}^{\times 6} \widetilde{\rho}_{k}^{*}\left(\widetilde{\lambda}_{1} \widetilde{\mu}_{1}\right)+\sum_{l \neq k} \pi_{*}^{\times 6}\left(\widetilde{\rho}_{k}^{*} \widetilde{\lambda}_{1} \widetilde{\rho}_{l}^{*} \widetilde{\mu}_{1}\right)\right) \\
& =\sum_{k=1}^{6}\left(32 \rho_{k}^{*}\left(\lambda_{1} \mu_{1}\right)+16 \sum_{l \neq k} \rho_{k}^{*}\left(2 \lambda_{1}\right) \rho_{l}^{*} \mu_{1}\right) \\
& =32 \sum_{k=1}^{6} \sum_{l=1}^{6} \rho_{k}^{*} \lambda_{1} \rho_{l}^{*} \mu_{1} .
\end{aligned}
$$

The calculation for $\pi_{*}^{(6)} \sigma_{i}^{ \pm}=16\left(\eta+\sigma_{i}\right)$ is almost the same, but the terms with $l=k$ and $l \neq k$ have different coefficients (since $2 \lambda_{1}$ becomes $\lambda_{i}$ ). The sum of the $l \neq k$ terms with $\frac{1}{2}$ times the $l=k$ terms descends to $16 \sigma_{i}$. We are left with

$$
16 \sum_{k=1}^{6} \rho_{k}^{*}\left(\lambda_{i} \mu_{i}\right)
$$

which descends to $16 \eta$. Adding these up for all $i$ gives the required formula for $\pi_{*}^{(6)} \widetilde{\theta}$.

The projection formula implies that $\pi^{*}\left(\lambda_{1} \mu_{1}\right)=2 \widetilde{\lambda}_{1} \widetilde{\mu}_{1}$, so $\pi^{(6) *} \eta=2 \widetilde{\eta}$ by definition. Therefore

$$
\pi_{*}^{(6)}\left(2 \widetilde{\eta}^{2}\right)=\pi_{*}^{(6)}\left(\widetilde{\eta} \pi^{(6) *} \eta\right)=32 \eta^{2}
$$

giving the desired formula for $\pi_{*}^{(6)}\left(\widetilde{\eta}^{2}\right)$. The calculation for $\pi_{*}^{(6)}(\widetilde{\eta} \widetilde{\theta})$ is similar.

Computing $\pi_{*}^{(6)}\left(\widetilde{\theta}^{2}\right)$ is more complicated. If $i \neq j$ then $\sigma_{i}^{ \pm} \sigma_{j}^{ \pm}$corresponds to

$$
\sum_{k=1}^{6} \sum_{l=1}^{6} \sum_{m=1}^{6} \sum_{n=1}^{6} \widetilde{\rho}_{k}^{*} \lambda_{i}^{ \pm} \widetilde{\rho}_{l}^{*} \mu_{i}^{ \pm} \widetilde{\rho}_{m}^{*} \lambda_{j}^{ \pm} \widetilde{\rho}_{n}^{*} \mu_{j}^{ \pm}
$$

Let $\widetilde{S}$ be the sum of the terms in (8.1) whose indices are distinct. Also let $\widetilde{T}, \widetilde{U}$ and $\widetilde{V}$ the sums of those for which $k=l, m=n$, or both (with all the other pairs distinct). The remaining terms of (8.1) all vanish (e.g. when $k=m$, such a term contains $\left.\rho_{k}^{*}\left(\lambda_{i}^{ \pm} \lambda_{j}^{ \pm}\right)=0\right)$, so $\widetilde{S}+\widetilde{T}+\widetilde{U}+\widetilde{V}$ descends to $\sigma_{i}^{ \pm} \sigma_{j}^{ \pm}$. Moreover, for degree reasons

$$
\widetilde{V}=\sum_{k=1}^{6} \sum_{m \neq k} \widetilde{\rho}_{k}^{*}\left(\lambda_{i}^{ \pm} \mu_{i}^{ \pm}\right) \widetilde{\rho}_{m}^{*}\left(\lambda_{j}^{ \pm} \mu_{j}^{ \pm}\right)=\sum_{k=1}^{6} \sum_{m=1}^{6} \widetilde{\rho}_{k}^{*}\left(\lambda_{i}^{ \pm} \mu_{i}^{ \pm}\right) \widetilde{\rho}_{m}^{*}\left(\lambda_{j}^{ \pm} \mu_{j}^{ \pm}\right)
$$

descends to $\widetilde{\eta}^{2}$. Similarly, $\widetilde{T}+\widetilde{V}$ and $\widetilde{U}+\widetilde{V}$ correspond to $\widetilde{\eta} \sigma_{j}^{ \pm}$and $\sigma_{i}^{ \pm} \widetilde{\eta}$ respectively.

Define $S, T, U, V \in H^{4}\left(X^{(6)}, \mathbb{Z}\right)$ in an analogous way. Since each term in $\widetilde{S}$ involves four distinct projections (with two missing), $\pi_{*}^{\times 6} \widetilde{S}=4 S$. Applying this reasoning to the other sums gives

$$
\pi_{*}^{\times 6}(\widetilde{S}+\widetilde{T}+\widetilde{U}+\widetilde{V})=4 S+8(T+U)+16 V=4((S+T+U+V)+(T+V)+(U+V)+V)
$$

and hence

$$
\pi_{*}^{(6)}\left(\sigma_{i}^{ \pm} \sigma_{j}^{ \pm}\right)=4\left(\sigma_{i} \sigma_{j}+\eta \sigma_{j}+\sigma_{i} \eta+\eta^{2}\right)=4\left(\eta+\sigma_{i}\right)\left(\eta+\sigma_{j}\right) .
$$


The same formula clearly works for $\sigma_{i}^{ \pm} \sigma_{j}^{\mp}$. However, it breaks down for $\sigma_{i}^{ \pm} \sigma_{i}^{\mp}$, because the corresponding class in $H^{4}\left(X^{6}, \mathbb{Z}\right.$ ) has extra terms with $k=n$ and $l=m$ (so $S+T+U+V$ does not correspond to $\sigma_{i}^{2}$ ). In this case (i.e., when $i=j$ ), swapping $k$ and $m$ takes each term of $S$ to its negative, so $S=0$. On the other hand, $T+V$ and $U+V$ both correspond to $\eta \sigma_{i}$. It follows that

$$
\pi_{*}^{(6)}\left(\sigma_{i}^{ \pm} \sigma_{i}^{\mp}\right)=8 \eta \sigma_{i}+8 \eta \sigma_{i}=16 \eta \sigma_{i},
$$

because it corresponds to $8(T+U)+16 V=8(T+V)+8(U+V)$.

The calculation for $\widetilde{\sigma}_{1} \sigma_{j}^{ \pm}$is also a little different, because $S$ and $U$ pick up a factor of two when pushing forward $\widetilde{\lambda}_{1}$ ( $T$ and $V$ do not because they involve $\widetilde{\lambda}_{1} \widetilde{\mu}_{1}$ instead). In other words

$$
\pi_{*}^{\times 6}(\widetilde{S}+\widetilde{T}+\widetilde{U}+\widetilde{V})=8(S+T)+16(U+V)=8((S+T+U+V)+(U+V))
$$

and hence

$$
\pi_{*}^{(6)}\left(\widetilde{\sigma}_{1} \sigma_{j}^{ \pm}\right)=8\left(\sigma_{1} \sigma_{j}+\sigma_{1} \eta\right)=8 \sigma_{1}\left(\eta+\sigma_{j}\right) .
$$

The formula for $\pi_{*}^{(6)}\left(\widetilde{\theta}^{2}\right)$ can be found by adding all of these terms.

Remark 8.2. The general formula, for a curve $X$ of genus $g$, is

$$
\pi_{*}^{(d)}\left(\widetilde{\eta}^{p} \widetilde{\theta}^{q}\right)=2^{d-p-q} \sum_{l=0}^{q}\left(\begin{array}{c}
g-1 \\
q-l
\end{array}\right) \frac{q !}{l !} \eta^{p+q-l} \theta^{l} .
$$

This can be proved by generalizing the above argument (see [Con19] for details).

8.2. The ranks of $\mathbb{K}^{+}$and $\mathbb{K}^{-}$. Although the results of this Paragraph follow from Paragraph 8.3, we feel that it is worth including because the proof here is considerably simpler than the general calculation of Hodge numbers in Paragraph 8.3 and also provides an independent verification of Paragraph 8.3.

We know that $\Theta \hookrightarrow A$ satisfies the Lefschetz hyperplane theorem for $H^{*}(-, \mathbb{Q})$. Since pullback and Gysin maps on cohomology are $(-1)$-equivariant, the theorem also holds for $H^{*}(-, \mathbb{Q})^{+}$:

Proposition 8.3. (1) For $n<g-1, H^{n}(\Theta, \mathbb{Q})^{+} \leftarrow H^{n}(A, \mathbb{Q})^{+}$is an isomorphism.

(2) For $g-1<n \leq 2 g-2, H^{n}(\Theta, \mathbb{Q})^{+} \rightarrow H^{n+2}(A, \mathbb{Q})^{+}$is an isomorphism.

(3) For $n=g-1$, the pullback $H^{g-1}(A, \mathbb{Q})^{+} \rightarrow H^{g-1}(\Theta, \mathbb{Q})^{+}$is an injection.

Let $A[2]$ be the set of 2-torsion points of $A$, and put $\Theta[2]:=A[2] \cap \Theta$. We have $\# A[2]=2^{2 g}$ and, since $\Theta$ is smooth, $\# \Theta[2]=2^{g-1}\left(2^{g}-1\right)$. Also let $A \stackrel{\pi}{\rightarrow} A^{+}$and $\Theta \stackrel{\pi}{\rightarrow} \Theta^{+}$be the quotients of $A$ and $\Theta$ by -1 .

Lemma 8.4. $\chi\left(A^{+}\right)=2^{2 g-1}$ and $\chi\left(\Theta^{+}\right)=\frac{1}{2}(-1)^{g-1} g !+2^{g-2}\left(2^{g}-1\right)$ 
Proof. It is well-known that $\chi(A)=0$ and $\chi(\Theta)=(-1)^{g-1} g$ !. Since $A$ is a double cover of $A^{+}$ ramified at $2^{2 g}$ points, $\chi(A)=2 \chi\left(A^{+}\right)-2^{2 g}$ and hence $\chi\left(A^{+}\right)=2^{2 g-1}$. Similarly

$$
\chi\left(\Theta^{+}\right)=\frac{1}{2}(\chi(\Theta)+\# \Theta[2])=\frac{1}{2}(-1)^{g-1} g !+2^{g-2}\left(2^{g}-1\right) .
$$

Proposition 8.5. We have

$$
\operatorname{dim} \frac{H^{g-1}(\Theta, \mathbb{Q})^{+}}{H^{g-1}(A, \mathbb{Q})^{+}}=\frac{g !}{2}+(-1)^{g} 2^{g-2}\left(2^{g}+1\right)+\left\{\begin{array}{cc}
-\left(\begin{array}{c}
2 g \\
g
\end{array}\right) & g \text { even } \\
\left(\begin{array}{c}
2 g \\
g+1
\end{array}\right) & g \text { odd }
\end{array}\right.
$$

Proof. By [Mac62, (1.2)] and the injectivity of Proposition 8.3,

$$
\operatorname{dim} \frac{H^{g-1}(\Theta, \mathbb{Q})^{+}}{H^{g-1}(A, \mathbb{Q})^{+}}=h^{g-1}\left(\Theta^{+}\right)-h^{g-1}\left(A^{+}\right) .
$$

We have $2\left(\chi\left(A^{+}\right)-\chi\left(\Theta^{+}\right)\right)=\# A[2]-\# \Theta[2]+(-1)^{g} g !=2^{g-1}\left(2^{g}+1\right)+(-1)^{g} g$ !. By Proposition 8.3, we can match up all cohomology groups of $\Theta^{+}$with isomorphic cohomology groups of $A^{+}$, with the exception of degree $g-1$ on the $\Theta^{+}$side and degrees $g-1, g, g+1$ on the $A^{+}$side. Thus

$$
\chi\left(A^{+}\right)-\chi\left(\Theta^{+}\right)=(-1)^{g}\left(h^{g-1}\left(\Theta^{+}\right)-h^{g-1}\left(A^{+}\right)+h^{g}\left(A^{+}\right)-h^{g+1}\left(A^{+}\right)\right),
$$

which means

$$
h^{g-1}\left(\Theta^{+}\right)-h^{g-1}\left(A^{+}\right)=(-1)^{g} \frac{2^{g-1}\left(2^{g}+1\right)+(-1)^{g} g !}{2}+(-1)^{g}\left(h^{g+1}\left(A^{+}\right)-h^{g}\left(A^{+}\right)\right) .
$$

Finally note that $h^{n}\left(A^{+}\right)=0$ for $n$ odd and $h^{n}\left(A^{+}\right)=\left(\begin{array}{c}2 g \\ n\end{array}\right)$ for $n$ even.

\section{Proposition 8.6.}

$$
\begin{array}{r}
\operatorname{dim} \mathbb{K}_{\mathbb{Q}}^{-}=\frac{g !}{2}+(-1)^{g-1}\left(2^{g-2}\left(2^{g}+1\right)\right)+\left\{\begin{array}{cc}
\left(\begin{array}{c}
2 g \\
g+1
\end{array}\right) & g \text { even } \\
-\left(\begin{array}{c}
2 g \\
g
\end{array}\right) & g \text { odd }
\end{array}\right. \\
\operatorname{dim} \mathbb{K}_{\mathbb{Q}}^{+}=\frac{g !}{2}+(-1)^{g}\left(2^{g-2}\left(2^{g}+1\right)\right)+\left\{\begin{array}{cc}
-\left(\begin{array}{c}
2 g \\
g
\end{array}\right) & g \text { even } \\
\left(\begin{array}{c}
2 g \\
g+1
\end{array}\right) & g \text { odd }
\end{array}\right.
\end{array}
$$

Proof. Taking -1 invariants of the exact sequence

$$
0 \rightarrow H^{g-1}(A, \mathbb{Q}) \rightarrow H^{g-1}(\Theta, \mathbb{Q}) \rightarrow \mathbb{K}_{\mathbb{Q}} \rightarrow 0
$$

gives an isomorphism between the -1 invariant component of $\mathbb{K}$ and $\frac{H^{g-1}(\Theta, \mathbb{Q})^{+}}{H^{g-1}(A, \mathbb{Q})^{+}}$. On the other hand, $\operatorname{dim} \mathbb{K}_{\mathbb{Q}}=g !+\left(\begin{array}{c}2 g \\ g+1\end{array}\right)-\left(\begin{array}{c}2 g \\ g\end{array}\right)$. After subtracting and using the previous formula we get the statement. 


\subsection{The Hodge numbers of $\mathbb{K}^{+}$and $\mathbb{K}^{-}$.}

Proposition 8.7. The Hodge numbers are

$$
h^{p, g-1-p}(\mathbb{K})=\left\langle\begin{array}{l}
g \\
p
\end{array}\right\rangle-\left(\begin{array}{c}
g \\
p
\end{array}\right)\left(\begin{array}{c}
g-1 \\
p
\end{array}\right)+\left(\begin{array}{c}
g \\
p+1
\end{array}\right)\left(\begin{array}{l}
g-1 \\
p-1
\end{array}\right),
$$

where $\left\langle\begin{array}{c}g \\ p\end{array}\right\rangle:=\sum_{k=0}^{p}\left(\begin{array}{c}g+1 \\ k\end{array}\right)(-1)^{k}(p+1-k)^{g}$ is an eulerian number (see [GKP94, (6.38)]).

Proof. The Hodge number $h^{p, g-1-p}(\mathbb{K})$ appears in

$$
\begin{aligned}
\chi\left(\Omega_{\Theta}^{p}\right) & =\sum_{k=0}^{g-1}(-1)^{k} h^{p, k}(\Theta) \\
& =\sum_{k=0}^{g-1-p}(-1)^{k} h^{p, k}(A)+(-1)^{g-1-p} h^{p, g-1-p}(\mathbb{K})+\sum_{k=g-p}^{g-1}(-1)^{k} h^{p+1, k+1}(A) .
\end{aligned}
$$

Using a standard identity [GKP94, (5.16)], the first term simplifies to

$$
\sum_{k=0}^{g-1-p}(-1)^{k}\left(\begin{array}{l}
g \\
p
\end{array}\right)\left(\begin{array}{l}
g \\
k
\end{array}\right)=(-1)^{g-1-p}\left(\begin{array}{c}
g \\
p
\end{array}\right)\left(\begin{array}{c}
g-1 \\
p
\end{array}\right),
$$

and similarly $\sum_{k=g-p}^{g-1}(-1)^{k} h^{p+1, k+1}(A)=(-1)^{g-p}\left(\begin{array}{c}g \\ p+1\end{array}\right)\left(\begin{array}{c}g-1 \\ p-1\end{array}\right)$. Next, we will compute $\chi\left(\Omega_{\Theta}^{p}\right)$. The conormal bundle sequence for $\Theta \subset A$ induces exact sequences

$$
\left.0 \rightarrow \Omega_{\Theta}^{p-1}(-\Theta) \rightarrow \Omega_{A}^{p}\right|_{\Theta} \rightarrow \Omega_{\Theta}^{p} \rightarrow 0
$$

for all $p \in\{1, \ldots, g\}$, so by induction (and the triviality of $\Omega_{A}$ )

$$
\operatorname{ch}\left(\Omega_{\Theta}^{p}\right)=\left.\sum_{k=0}^{p}\left(\begin{array}{l}
g \\
k
\end{array}\right)\left(-e^{-\theta}\right)^{p-k}\right|_{\Theta}
$$

Moreover $\operatorname{td}(\Theta)=\operatorname{td}\left(\mathcal{O}_{\Theta}(\Theta)\right)^{-1}=\left.\frac{1-e^{-\theta}}{\theta}\right|_{\Theta}$, and hence

$$
\begin{aligned}
\chi\left(\Omega_{\Theta}^{p}\right) & =\int_{\Theta} \operatorname{ch}\left(\Omega_{\Theta}^{p}\right) \operatorname{td}(\Theta) \\
& =\int_{A} \sum_{k=0}^{p}\left(\begin{array}{l}
g \\
k
\end{array}\right)\left(-e^{-\theta}\right)^{p-k}\left(1-e^{-\theta}\right) \\
& =\int_{A}\left(\begin{array}{c}
p \\
k=0
\end{array}\left(\begin{array}{c}
g+1 \\
k
\end{array}\right)\left(-e^{-\theta}\right)^{p+1-k}+\left(\begin{array}{c}
g \\
p
\end{array}\right)\right) \\
& =\sum_{k=0}^{p}\left(\begin{array}{c}
g+1 \\
k
\end{array}\right)(-1)^{p+1-k}(k-p-1)^{g},
\end{aligned}
$$

since $\int_{A} \frac{\theta^{g}}{g !}=\chi\left(\mathcal{O}_{A}(\Theta)\right)=1$. After some rearranging, this gives the required formula. 
A similar argument can be used to compute the Hodge numbers of $\mathbb{K}^{+}$, as follows. Let $\widetilde{\Theta} \stackrel{\beta}{\rightarrow} \Theta$ be the blowup of $\Theta$ at its 2-torsion points, with $\Delta \subset \widetilde{\Theta}$ the exceptional divisor. If $\widetilde{\Theta} \stackrel{\pi}{\rightarrow} \widetilde{\Theta}^{+}$is the quotient of $\widetilde{\Theta}$ by the induced action of -1 , then $\pi^{*}: H^{*}(\widetilde{\Theta}, \mathbb{Q}) \rightarrow H^{*}(\widetilde{\Theta}, \mathbb{Q})^{+}$is an isomorphism $\left[\right.$ Mac62, (1.2)]. Set $\mathcal{O}_{\widetilde{\Theta}}(1):=\mathcal{O}_{\widetilde{\Theta}}(-\Delta)$. For each $p \in \mathbb{N}$ there are exact sequences

$$
0 \rightarrow\left(\beta^{*} \Omega_{\Theta}^{p}\right)(1-p) \rightarrow \Omega_{\widetilde{\Theta}}^{p} \rightarrow \Omega_{\Delta}^{p} \rightarrow 0
$$

and

$$
0 \rightarrow \pi^{*} \Omega_{\widetilde{\Theta}^{+}}^{p+1} \rightarrow \Omega_{\widetilde{\Theta}}^{p+1} \rightarrow \Omega_{\Delta}^{p}(1) \rightarrow 0 .
$$

It is straightforward to verify this by local calculations (for coordinate-free proofs, see [Con19, §1.2]). The following "dual" sequences will also be useful:

$$
0 \rightarrow T_{\widetilde{\Theta}} \rightarrow \beta^{*} T_{\Theta} \rightarrow T_{\Delta}(-1) \rightarrow 0
$$

and

$$
0 \rightarrow T_{\widetilde{\Theta}} \rightarrow \pi^{*} T_{\widetilde{\Theta}^{+}} \rightarrow \mathcal{O}_{\Delta}(-2) \rightarrow 0 .
$$

Proposition 8.8. The Hodge numbers $h^{p, g-1-p}\left(\mathbb{K}^{+}\right)$of $\mathbb{K}^{+}$are

$$
\frac{1}{2}\left\langle\begin{array}{l}
g \\
p
\end{array}\right\rangle+(-1)^{g}\left(\left(\begin{array}{l}
g \\
p
\end{array}\right) \sum_{q=0}^{g-1-p}\left(\begin{array}{l}
g \\
q
\end{array}\right) \epsilon_{p+q}+\left(\begin{array}{c}
g \\
p+1
\end{array}\right) \sum_{q=g-p}^{g-1}\left(\begin{array}{c}
g \\
q+1
\end{array}\right) \epsilon_{p+q}-\left(\begin{array}{c}
g-1 \\
p
\end{array}\right) \frac{2^{g}-1}{2}\right),
$$

where $\epsilon_{k}:=\frac{1}{2}\left(1+(-1)^{k}\right)$ is one (resp. zero) if $k$ is even (resp. odd).

Proof. If $p=0$ then $h^{p, g-1-p}\left(\mathbb{K}^{+}\right)=h^{p, g-1-p}(\mathbb{K})=0$. Otherwise, by Hirzebruch-Riemann-Roch and the above sequences

$$
\begin{aligned}
2 \chi\left(\Omega_{\widetilde{\Theta}^{+}}^{p}\right) & =2 \int_{\widetilde{\Theta}^{+}} \operatorname{ch}\left(\Omega_{\widetilde{\Theta}^{+}}^{p}\right) \operatorname{td}\left(\widetilde{\Theta}^{+}\right) \\
& =\int_{\widetilde{\Theta}} \pi^{*}\left(\operatorname{ch}\left(\Omega_{\widetilde{\Theta}^{+}}^{p}\right) \operatorname{td}\left(\widetilde{\Theta}^{+}\right)\right) \\
& =\int_{\widetilde{\Theta}}\left(\operatorname{ch}\left(\Omega_{\widetilde{\Theta}}^{p}\right)-\operatorname{ch}\left(\Omega_{\Delta}^{p-1}(1)\right)\right) \operatorname{td}(\widetilde{\Theta}) \operatorname{td}\left(\mathcal{O}_{\Delta}(-2)\right) \\
& =\int_{\widetilde{\Theta}}\left(\operatorname{ch}\left(\beta^{*} \Omega_{\Theta}^{p}\right) \operatorname{ch}\left(\mathcal{O}_{\widetilde{\Theta}}(1-p)\right)+\operatorname{ch}\left(\Omega_{\Delta}^{p}\right)-\operatorname{ch}\left(\Omega_{\Delta}^{p-1}(1)\right)\right) \operatorname{td}(\widetilde{\Theta}) \operatorname{td}\left(\mathcal{O}_{\Delta}(-2)\right)
\end{aligned}
$$

The first term can be computed on $\Theta$. To be specific:

$$
\begin{aligned}
\chi_{1} & :=\int_{\widetilde{\Theta}} \operatorname{ch}\left(\beta^{*} \Omega_{\Theta}^{p}\right) \operatorname{ch}\left(\mathcal{O}_{\widetilde{\Theta}}(1-p)\right) \operatorname{td}(\widetilde{\Theta}) \operatorname{td}\left(\mathcal{O}_{\Delta}(-2)\right) \\
& =\int_{\Theta} \operatorname{ch}\left(\Omega_{\Theta}^{p}\right) \operatorname{td}(\Theta) \cdot \beta_{*}\left(\operatorname{ch}\left(\mathcal{O}_{\widetilde{\Theta}}(1-p)\right) \frac{\operatorname{td}\left(\mathcal{O}_{\Delta}(-2)\right)}{\operatorname{td}\left(T_{\Delta}(-1)\right)}\right)
\end{aligned}
$$


The Euler sequence and the ideal sheaf sequence for $\Delta \subset \widetilde{\Theta}$ imply that

$$
\operatorname{td}\left(T_{\Delta}(-1)\right)=\frac{\operatorname{td}\left(\mathcal{O}_{\Delta}\right)^{g-1}}{\operatorname{td}\left(\mathcal{O}_{\Delta}(-1)\right)}=\frac{\operatorname{td}\left(\mathcal{O}_{\widetilde{\Theta}}(1)\right)^{1-g}}{\operatorname{td}\left(\mathcal{O}_{\widetilde{\Theta}}(-1)\right)}
$$

and similarly $\operatorname{td}\left(\mathcal{O}_{\Delta}(-2)\right)=\operatorname{td}\left(\mathcal{O}_{\widetilde{\Theta}}(-2)\right) \operatorname{td}\left(\mathcal{O}_{\widetilde{\Theta}}(-1)\right)^{-1}$, so

$$
\chi_{1}=\int_{\Theta} \operatorname{ch}\left(\Omega_{\Theta}^{p}\right) \operatorname{td}(\Theta) \cdot \beta_{*}\left(\operatorname{ch}\left(\mathcal{O}_{\widetilde{\Theta}}(1-p)\right) \operatorname{td}\left(\mathcal{O}_{\widetilde{\Theta}}(1)\right)^{g-1} \operatorname{td}\left(\mathcal{O}_{\widetilde{\Theta}}(-2)\right)\right) .
$$

Everything inside $\beta_{*}$ is a polynomial in $h:=c_{1}\left(\mathcal{O}_{\widetilde{\Theta}}(1)\right)$. Since $\beta$ contracts $\Delta$ the only powers of $h$ which survive are $h^{0}$ and $h^{g-1}$. Therefore

$$
\chi_{1}=\chi\left(\Omega_{\Theta}^{p}\right)-\left(\begin{array}{c}
g-1 \\
p
\end{array}\right) \int_{\tilde{\Theta}} e^{(1-p) h}\left(\frac{h}{1-e^{-h}}\right)^{g-1} \frac{2 h}{1-e^{2 h}} .
$$

Since $-h$ is the class of $\Delta \subset \widetilde{\Theta}$, which has $2^{g-1}\left(2^{g}-1\right)$ components,

$$
\chi_{1}=\chi\left(\Omega_{\Theta}^{p}\right)-\left(\begin{array}{c}
g-1 \\
p
\end{array}\right) 2^{g-1}\left(2^{g}-1\right) \chi_{2},
$$

where $\chi_{2}$ is the residue of

$$
-\frac{e^{(1-p) z}}{\left(1-e^{-z}\right)^{g-1}} \cdot \frac{2}{1-e^{2 z}}=-\frac{e^{(g-p) z}}{\left(e^{z}-1\right)^{g-1}} \cdot \frac{2}{\left(1-e^{z}\right)\left(1+e^{z}\right)}=\frac{2 e^{(g-p) z}}{\left(e^{z}-1\right)^{g}\left(e^{z}+1\right)}
$$

at 0 , or equivalently (making the change of variables $w:=e^{z}-1$ ), the residue of

$$
\frac{2(w+1)^{g-1-p}}{w^{g}(w+2)}
$$

at 0 . Since $\frac{2}{w+2}=\sum_{k=0}^{\infty}\left(-\frac{w}{2}\right)^{k}$, taking the Laurent expansion of (8.2) gives

$$
\chi_{2}=\sum_{k=0}^{g-1-p}\left(\begin{array}{c}
g-1-p \\
i
\end{array}\right)\left(-\frac{1}{2}\right)^{g-1-i}=\left(-\frac{1}{2}\right)^{p}\left(1-\frac{1}{2}\right)^{g-1-p}=\frac{(-1)^{p}}{2^{g-1}} .
$$

Grothendieck-Riemann-Roch allows us to compute the remaining terms on $\Delta$ :

$$
\begin{aligned}
\chi_{3} & :=\int_{\widetilde{\Theta}}\left(\operatorname{ch}\left(\Omega_{\Delta}^{p}\right)-\operatorname{ch}\left(\Omega_{\Delta}^{p-1}(1)\right)\right) \operatorname{td}(\widetilde{\Theta}) \frac{\operatorname{td}\left(\mathcal{O}_{\widetilde{\Theta}}(-2)\right)}{\operatorname{td}\left(\mathcal{O}_{\widetilde{\Theta}}(-1)\right)} \\
& =\int_{\Delta}\left(\operatorname{ch}\left(\Omega_{\Delta}^{p}\right)-\operatorname{ch}\left(\Omega_{\Delta}^{p-1}(1)\right)\right) \operatorname{td}(\Delta) \frac{\operatorname{td}\left(\mathcal{O}_{\Delta}(-2)\right)}{\operatorname{td}\left(\mathcal{O}_{\Delta}(-1)\right)} .
\end{aligned}
$$

The following exact sequence arises from the Euler sequence on $\Delta$ :

$$
0 \rightarrow \Omega_{\Delta}^{p} \rightarrow \mathcal{O}_{\Delta}(-p)^{\oplus\left(\begin{array}{c}
g-1 \\
p
\end{array}\right)} \rightarrow \Omega_{\Delta}^{p-1} \rightarrow 0 .
$$

It implies that $\operatorname{ch}\left(\Omega_{\Delta}^{p}\right)=\left(\begin{array}{c}g-1 \\ p\end{array}\right) \operatorname{ch}\left(\mathcal{O}_{\Delta}(-p)\right)-\operatorname{ch}\left(\Omega_{\Delta}^{p-1}\right)$, so by induction

$$
\operatorname{ch}\left(\Omega_{\Delta}^{p}\right)=\sum_{k=0}^{p}(-1)^{p-k}\left(\begin{array}{c}
g-1 \\
k
\end{array}\right) e^{-k h}
$$


where $h:=c_{1}\left(\mathcal{O}_{\Delta}(1)\right)$. Since

$$
\frac{\operatorname{td}\left(\mathcal{O}_{\Delta}(-2)\right)}{\operatorname{td}\left(\mathcal{O}_{\Delta}(-1)\right)}=\frac{2 h}{1-e^{2 h}} \cdot \frac{1-e^{h}}{h}=\frac{2}{e^{h}+1},
$$

it follows that $\chi_{3}=2^{g-1}\left(2^{g}-1\right) \chi_{4}$, where $\chi_{4}$ is the residue of

$$
\begin{aligned}
& \left(\sum_{k=0}^{p}(-1)^{p-k}\left(\begin{array}{c}
g-1 \\
k
\end{array}\right) e^{-k z}+\sum_{k=0}^{p-1}(-1)^{p-k}\left(\begin{array}{c}
g-1 \\
k
\end{array}\right) e^{(1-k) z}\right) \frac{2}{\left(1-e^{-z}\right)^{g-1}\left(e^{z}+1\right)} \\
= & \left(\sum_{k=0}^{p}(-1)^{p-k}\left(\begin{array}{c}
g-1 \\
k
\end{array}\right) e^{(g-1-k) z}+\sum_{k=0}^{p-1}(-1)^{p-k}\left(\begin{array}{c}
g-1 \\
k
\end{array}\right) e^{(g-k) z}\right) \frac{2}{\left(e^{z}-1\right)^{g-1}\left(e^{z}+1\right)}
\end{aligned}
$$

at 0 . The above residue calculations, with some minor adjustments for the extremal terms in the sums, can be used to show that

$$
\chi_{4}=\sum_{k=0}^{p} \frac{(-1)^{p}}{2^{g-2}}\left(\begin{array}{c}
g-1 \\
k
\end{array}\right)-\sum_{k=0}^{p-1} \frac{(-1)^{p}}{2^{g-2}}\left(\begin{array}{c}
g-1 \\
k
\end{array}\right)+2(-1)^{p} \delta_{p}=\frac{(-1)^{p}}{2^{g-2}}\left(\begin{array}{c}
g-1 \\
p
\end{array}\right)+2(-1)^{p} \delta_{p},
$$

where $\delta_{0}:=0, \delta_{g-1}=0$ and $\delta_{p}:=1$ for $0<p<g-1$. Therefore

$$
\begin{aligned}
2 \chi\left(\Omega_{\widetilde{\Theta}^{+}}^{p}\right) & =\chi_{1}+\chi_{3} \\
& =\chi\left(\Omega_{\Theta}^{p}\right)-(-1)^{p}\left(\begin{array}{c}
g-1 \\
p
\end{array}\right)\left(2^{g}-1\right)+(-1)^{p}\left(\begin{array}{c}
g-1 \\
p
\end{array}\right) 2\left(2^{g}-1\right)+(-1)^{p} 2^{g}\left(2^{g}-1\right) \delta_{p} \\
& =\chi\left(\Omega_{\Theta}^{p}\right)+(-1)^{p}\left(2^{g}-1\right)\left(\left(\begin{array}{c}
g-1 \\
p
\end{array}\right)+2^{g} \delta_{p}\right) .
\end{aligned}
$$

Decomposing $H^{*}(\widetilde{\Theta}, \mathbb{Q})$ as in $[$ Bea77b, 0.1.3.ii] gives

$$
\begin{aligned}
\chi\left(\Omega_{\widetilde{\Theta}^{+}}^{p}\right) & =\sum_{q=0}^{g-1}(-1)^{q} \operatorname{dim}\left(H^{p, q}(\widetilde{\Theta}, \mathbb{Q})^{+}\right) \\
& =\sum_{q=0}^{g-1}(-1)^{q} \operatorname{dim}\left(H^{p, q}(\Theta, \mathbb{Q})^{+}\right)+(-1)^{p} 2^{g-1}\left(2^{g}-1\right) \delta_{p} .
\end{aligned}
$$

Combining (8.3) and (8.4) allows us to express $(-1)^{g-1-p} h^{p, g-1-p}\left(\mathbb{K}^{+}\right)$as

$$
\frac{1}{2} \chi\left(\Omega_{\Theta}^{p}\right)+(-1)^{p} \frac{2^{g}-1}{2}\left(\begin{array}{c}
g-1 \\
p
\end{array}\right)-\sum_{q=0}^{g-1-p}(-1)^{q} h^{p, q}(A) \epsilon_{p+q}-\sum_{q=g-p}^{g-1}(-1)^{q} h^{p+1, q+1}(A) \epsilon_{p+q},
$$

as required.

\section{REFERENCES}

[ACG11] E. Arbarello, M. Cornalba, and P.A. Griffiths, Geometry of algebraic curves, volume II with a contribution by J.D. Harris, Springer, Berlin, 2011.

[ACGH85] E. Arbarello, M. Cornalba, P.A. Griffiths, and J.D. Harris, Geometry of algebraic curves, volume I, Springer, Berlin, 1985. 
[AFS15] E. Arbarello, A. Ferretti, and G. Sacca, Relative Prym varieties associated to the double cover of an Enriques surface, J. Differential Geom. 100 (2015), no. 2, 191-250.

[BD87] A. Beauville and O. Debarre, Sur le problème de Schottky pour les variétés de Prym, Ann. Scuola Norm. Sup. Pisa Cl. Sci. (4) 14 (1987), no. 4, 613-623.

[Bea77a] A. Beauville, Prym varieties and the Schottky problem, Inventiones Math. 41 (1977), 149-196.

[Bea77b] _ _ Variétés de Prym et jacobiennes intermédiaires, Annales Sc. de l'École Norm. Sup., 4ème série 10 (1977), 309-391.

[Bea82] _ Sous-variétés spéciales des variétés de Prym, Compositio Math. 45, Fasc. 3 (1982), 357-383.

[BL04] C. Birkenhake and H. Lange, Complex abelian varieties, Grundlehren der Mathematischen Wissenschaften [Fundamental Principles of Mathematical Sciences], vol. 302, Springer, Berlin, 2004.

[Blo94] S. Bloch, The moving lemma for higher Chow groups, J. Algebraic Geom. 3 (1994), no. 3, 537-568. MR 1269719

[Con19] J. Conder, Geometric links between exceptional root lattices and the cohomology of theta divisors, Ph.D. thesis, University of California, San Diego, June 2019.

[DH88] S. Diaz and J. Harris, Geometry of the Severi variety, Trans. Amer. Math. Soc. 309 (1988), no. 1, 1-34. MR 957060

[Dol12] I. Dolgachev, Classical algebraic geometry. a modern view, Cambridge University Press, Cambridge, UK, 2012.

[Don92] R. Donagi, The fibers of the Prym map, Curves, Jacobians, and Abelian Varieties (Amherst, MA 1990), Contemp. Math., vol. 136, Amer. Math. Soc., 1992, pp. 55-125.

[DS81] R. Donagi and R. Smith, The structure of the Prym map, Acta Math. 146 (1981), 25-102.

[Ful84] W. Fulton, Intersection theory, Springer, Berlin, 1984.

[GKP94] R. L. Graham, D. E. Knuth, and O. Patashnik, Concrete mathematics, a foundation for computer science, Addison-Wesley, New York, 1994.

[Har77] R. Hartshorne, Algebraic geometry, Springer, Berlin, 1977.

[IS95] E. Izadi and D. van Straten, The intermediate jacobians of the theta-divisors of four-dimensional principally polarized abelian varieties, Journal of Algebraic Geometry 4 (1995), no. 3, 557-590.

[ITW17] E. Izadi, Cs. Tamas, and J. Wang, The primitive cohomology of the theta divisor of an abelian fivefold, J. Algebraic Geom. 26 (2017), 107-175.

[IW15] E. Izadi and J. Wang, The primitive cohomology of theta divisors, Proceedings of the conference on Hodge theory and classical algebraic geometry, Contemp. Math., vol. 647, AMS, Providence, RI, 2015, pp. 79-90.

[IW18] E. Izadi and J. Wang, The irreducible components of the primal cohomology of the theta divisor of an abelian fivefold, Amer. J. Math. (To appear).

[Iza95] E. Izadi, The geometric structure of $\mathcal{A}_{4}$, the structure of the Prym map, double solids and $\Gamma_{00}$-divisors, Journal für die Reine und Angewandte Mathematik 462 (1995), 93-158.

[Krä15] T. Krämer, On a family of surfaces of general type attached to abelian four-folds and the Weyl group $W\left(E_{6}\right)$, Int. Math. Res. Not. IMRN (2015), no. 24, 13062-13105. MR 3436138

[Mac62] I. G. Macdonald, Symmetric products of an algebraic curve, Topology 1 (1962), 319-343.

[MM83] S. Mori and S. Mukai, The uniruledness of the moduli space of curves of genus 11, Algebraic geometry (Tokyo/Kyoto, 1982), Lecture Notes in Math., vol. 1016, Springer, Berlin, 1983, pp. 334-353. 
[Mum74] D. Mumford, Prym varieties I, Contributions to Analysis (L.V. Ahlfors, I. Kra, B. Maskit, and L. Niremberg, eds.), Academic Press, 1974, pp. 325-350.

[OS79] T. Oda and C. S. Seshadri, Compactifications of the generalized Jacobian variety, Trans. Amer. Math. Soc. 253 (1979), 1-90. MR 536936

[Rec74] S. Recillas, Jacobians of curves with a $g_{4}^{1}$ are Prym varieties of trigonal curves, Bol. Soc. Math. Mexicana 19 (1974), 9-13.

[Spa94] E. H. Spanier, Algebraic topology, Springer-Verlag, New York, [1994], Corrected reprint of the 1966 original. MR 1325242

[Wel81] G. E. Welters, Abel-Jacobi isogenies for certain types of fano threefolds, Mathematical Centre Tracts, CWI Tracts 141 (1981), 1-139.

[Wel85] _ A theorem of Gieseker-Petri type for Prym varieties, Ann. Sci. École Norm. Sup. (4) 18 (1985), no. 4, 671-683. MR 839690

Department of Mathematics, University of California San Diego, 9500 Gilman Drive \# 0112, La JoLLA, CA 92093-0112, USA

E-mail address: jconder@math.ucsd.edu, jonno.conder@gmail.com

Department of Mathematics, University of California San Diego, 9500 Gilman Drive \# 0112, La JoLlA, CA 92093-0112, USA

E-mail address: ehdewey@math.ucsd.edu, ed.dewey@gmail.com

Department of Mathematics, University of California San Diego, 9500 Gilman Drive \# 0112, La JoLLA, CA 92093-0112, USA

E-mail address: eizadi@math.ucsd.edu 